\title{
Mathematical Modeling of Spray Impingement and Film Formation on Pharmaceutical Tablets during Coating
}

\author{
Charalampos Christodoulou ${ }^{\mathrm{a}}$, Eva Sorensen ${ }^{\mathrm{a}}$, Salvador García-Muñoz ${ }^{\mathrm{b}}, \mathrm{Luca}_{\mathrm{Mazzei}}{ }^{\mathrm{a} \dagger}$ \\ ${ }^{a}$ Department of Chemical Engineering, University College London, Torrington Place, London WC1E 7JE, UK \\ ${ }^{b}$ Eli Lilly and Company, Lilly Research Laboratories, Indianapolis, IN 46285, USA
}

\begin{abstract}
The application of coating films is an important step in the manufacture of pharmaceutical tablets. Understanding the phenomena taking place during coating spray application provides important information that can be used to reduce the number of defective tablets and select the optimal conditions for the coating process. In this work, we investigate spray impact and film spreading on a tablet while this passes through the spray-zone in a rotating coating drum. To simulate spray impingement, we developed an one-dimensional (1D) spreading model that is based on the mechanical energy equation. We assumed the spray to be uniform and we divided it into arrays of droplets that impinge successively on the substrate orthogonally to its surface. In the mechanical energy equation that describes the coating spreading, we accounted for the rate of work done on the surface of the liquid coating film by the impinging droplets that leads to volume change (film spreading and thickness increase). The novel model we propose in this work can calculate the coating spreading rate and thickness. We implemented the mathematical model employing the gPROMS Modelbuilder platform. To study the effect of coating properties and process parameters on the film spreading rate and on the final liquid film thickness, we performed variance-based sensitivity analysis. The model predictions are in good agreement with experimental data found in the literature.
\end{abstract}

\section{Introduction}

Coating of tablet cores is one of the oldest manufacturing processes utilized by the pharmaceutical industry $[1,2]$. Tablets are coated for several reasons, including enhancement of appearance, taste masking, protection of sensitive ingredients and control of the Active Pharmaceutical Ingredient (API) release [3]. Due to the complexity of the spray coating process, problems are often encountered with the final product $[4,5]$. Some of the most common tablet defects are bridging, cracking, colour variations, roughness/orange-peel roughness, picking and sticking [3]. One main cause of such defects is failing to adopt the right values for the process parameters in the coating drum, such as spray mass flow rate or coater temperature and rotational speed [5].

To address the above issues, researchers have investigated the coating spray atomization process [6], as well as the deceleration and the evaporation of the generated droplets during their flight from the nozzle to the tablet bed $[3,7]$. By combining these spray models with a thermodynamic model that calculates air temperature and humidity inside the coating drum [8], one can estimate the droplet size and velocity before impact [9]. Recent work on coating behavior after impact on a tablet core has been mainly focused on single droplet cases $[9,10]$, and therefore the information concerning spray impact and film formation under coating process conditions is limited [2]. Moreover, general spray impingement models found in the literature are either empirical or based on computationally expensive Computation Fluid Dynamics (CFD) simulations.

Previous work aiming to numerically simulate spray impingement on impermeable solid substrates mainly relied on models derived from single droplet impact studies [11]. Models following this approach describe the spray impingement as a superposition of single droplet impacts. Roisman et al. [12] questioned this modeling strategy, because it neglects interactions between neighbouring spreading droplets, a limitation that makes these models insensitive to spray density. Nevertheless, information about single droplet impacts offers useful insight into the complex spray impingement process [13]. Additionally, using Volume-Of-Fluid (VOF) CFD simulations, in the appendix of this article we show that in the conditions investigated in this work droplet-droplet interactions on the tablet are negligible.

\footnotetext{
${ }^{\dagger}$ Corresponding author.Tel. +44(0)207679 4328; Email: l.mazzei@ucl.ac.uk
} 
The models for single droplet impingement fall into two categories: those considering impact on dry substrates and those considering impact on wetted surfaces or liquid films. The impingement of a single droplet on a dry substrate can have several outcomes: the droplet may deposit into a cylindrical or spherical-cap film, disintegrate (splash) into secondary droplets, or recede and potentially rebound $[12,13]$. In the literature, one can find a few predictive theoretical models that simulate the spreading, receding, splashing and rebounding after droplet impingement on dry walls [14-17]. For pharmaceutical coating, Shaari [18] and Bolleddula et al. [10] experimentally investigated single droplet spreading on tablets, while we developed a model predicting the behavior of Opadry coating droplets after impact on dry porous substrates [19].

As mentioned, in the literature there are also a few models describing the impingement of a single droplet on a wetted surface. Roisman et al. [12] and Yarin et al. [20] experimentally and theoretically investigated droplet impingement on liquid films covering rigid substrates. They found that droplets with low impact velocity and small size can deposit on the film surface or coalesce, whereas droplets with moderate and high impact velocities tend to form a crater at the impingement region that leads to splashing and even to film disintegration. Few researchers have proposed empirical splashing and disintegration criteria or developed models for predicting the outcome of droplet impact on liquid films of different thickness [21].

About spray impingement, obtaining accurate and detailed information through experiments or mathematical modeling is challenging [11]. In the literature, one can find experimental work concerning the interaction of spray droplets impinging on a wall, and about splashing and breakup [20, 22]. Moreira et al. [13] reviewed the aforementioned studies that were mainly focused on simultaneous and subsequent impacts of two droplets. Mundo et al. [23] modeled spray impingement as a superposition of single droplet collisions without considering liquid film formation and the film-droplet interactions. Roisman et al. [16] developed a model to estimate the velocity and shape of the uprising liquid film, accounting for droplet collisions on the substrate and the influence of droplet spacing, but not including predictions for possible break-up of the uprising film. One drawback of these models is that they do not calculate the final film thickness after spray impingement.

Concerning the prediction of film thickness after spray impact, Lee and Ryou [24] developed an empirical model aiming to predict the outcome of diesel spray impingement on a rigid wall. Recently, Kalantari and Tropea [21] conducted experiments and derived a semi-empirical relation for the film thickness. We used their detailed experimental data and semi-empirical relation to validate the model developed in this work. In general, research for pharmaceutical sprays is focused on droplet atomization and evaporation [5], and therefore we were unable to find previous work that deals in detail with coating spray impact on tablets. To conclude, spray impact models available in the literature are either based on empirical equations or on computationally expensive CFD simulations [13].

In this paper, we present a novel model (Section 2) that describes spray impact on tablets during film-coating, without requiring prior knowledge of the process via empirical relations. The model predicts the time required for the wetting of the entire tablet surface facing the spray, as well as the liquid film thickness, while taking into account coating (viscosity, density, surface tension) and spray (droplet size, velocity, mass flow rate) properties.

Compared with CFD simulations - which take hours or days to output results - our model yields solutions considerably faster (simulation time $<5$ s) without sacrificing accuracy significantly. This allowed us to perform variance-based sensitivity analysis to study the influence of process parameters on the coating spreading behavior. We validated the numerical results with experimental data from the literature (Section 3). With this work, we aim to provide insight into the process of coating application on pharmaceutical tablets and to assist in the selection of the appropriate values for the process parameters required to minimize the number of defective tablets. 


\section{Mathematical model}

Film coatings are generally applied on tablets by spraying a polymer solution or dispersion on their surface [2]. After impingement onto the tablet, the droplets spread on its dry surface. Based on the experimental work of Bolleddula et al. [10] for pharmaceutical coating droplets, we assumed that the droplets spread without disintegrating or rebounding after impact. The spreading of a droplet that impacts on a dry rigid substrate can be divided into two consecutive regimes [10]: during the first the spreading is inertia driven, while during the second the droplet continues to spread by capillarity.

Continuous droplet impingement leads to the expansion of the wetted area and the formation of a liquid film that eventually covers the entire surface of the tablet. Following the liquid film formation, the solvent (normally water) both evaporates and concurrently absorbs into the porous core of the tablet leaving behind a dry polymeric film on the tablet surface (Fig. 1). In this work, we investigate multiple droplet impingement and spreading on a pharmaceutical tablet while the tablet passes through the spray-zone of a rotating coating drum.

To model spray impingement, we assumed that the spray is uniform and we divided it into arrays of droplets that impinge successively on the substrate orthogonally to its surface (Fig. 2a). To simplify the problem, we assumed that over a time interval of magnitude $t_{i}=D_{0} / U_{0}$, where $D_{0}$ and $\mathrm{U}_{0}$ represent the average diameter and velocity of the droplets before impact, respectively, the droplets in the first array impinge and spread without being affected by the subsequent droplets. The value of $t_{i}$ has the same order of magnitude as the time scale of the inertia-driven spreading regime reported in the literature [10]. Since the capillarity spreading is much slower, becoming significant only after several seconds, it can be neglected. At any given time $t<t_{i}$, we estimated the wetted area by calculating the spreading of a single droplet of initial diameter $\mathrm{D}_{0}$ and impact velocity $\mathrm{U}_{0}$ (Fig. 2b) and by assuming that all the droplets of the first array behave independently and identically as they impact on the dry tablet surface.

After the impact of the first array (initial tablet wetting), we assumed that the wetted area is made up of disconnected films, one associated with each droplet. The impingement of subsequent droplets on the surface of these films increases their volume, making them spread further and raising their thickness. We considered the spreading to be completed when the wetted area becomes equal to the area of the tablet surface that is facing the spray. We assumed that solvent evaporation and absorption into the tablet core become significant after the tablet is no longer under the spray, and therefore we neglected drying and absorption phenomena in the current model. This assumption is based on experimental observations which show that absorption and film drying are significantly slower than coating spreading after spray impingement $[9,18]$.

In this section we describe our mathematical model; this is presented in two subsections: Section 2.1 concerns the impact of the first array of droplets on the dry tablet core surface (initial wetting stage) and Section 2.2 deals with subsequent droplet impingement and spreading (impingement on wetted surface stage).

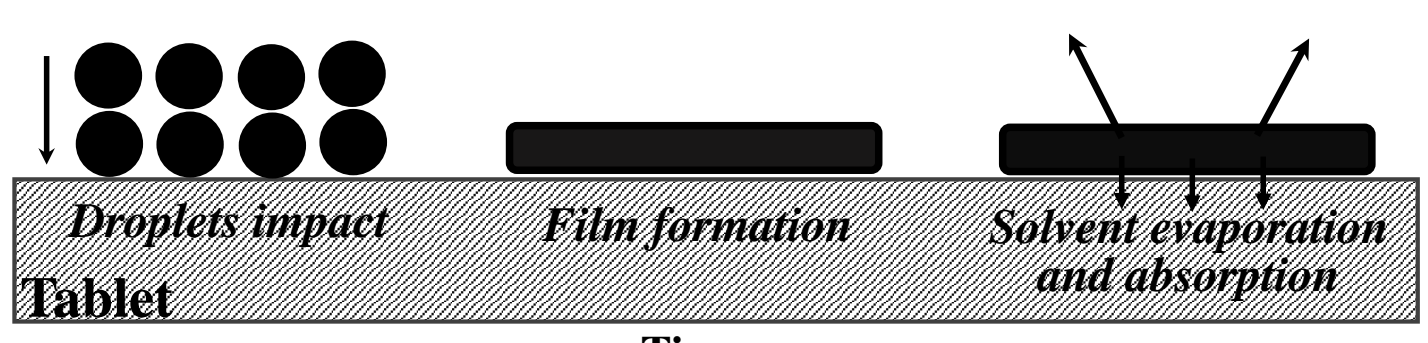

Time

Figure 1: Outline of the coating application process during pharmaceutical coating. 
(a)

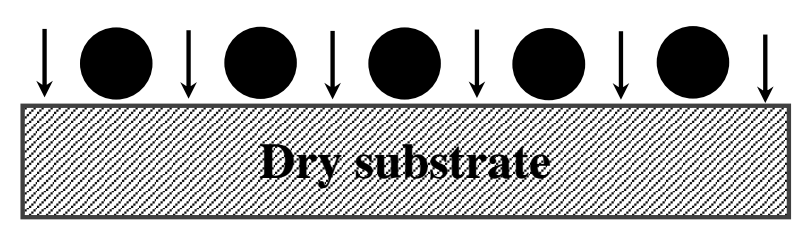

(b) Before impact After impact

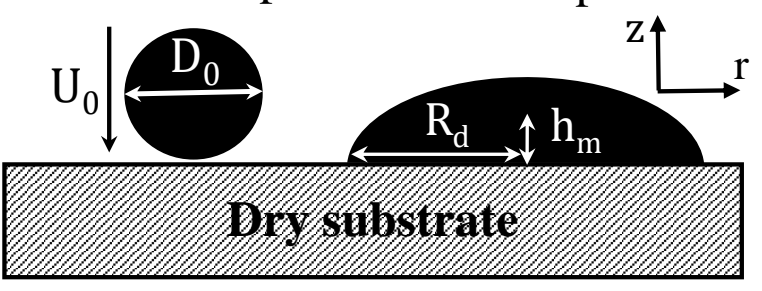

Figure 2: a) Droplet array and b) single droplet impact on a dry tablet.

\subsection{Initial wetting}

Here we present the part of the model that simulates multiple droplet impingement on dry surfaces of tablets entering the spray-zone. We assumed that all the droplets of the first array behave identically after impact since they are similarly sized and have similar impact velocities. This reasonable assumption allows us to simulate the spreading of a single droplet impacting the tablet with the average velocity and diameter of the spray droplets and then calculate, at any time $t<t_{i}$, the total wetted area on the tablet surface by multiplying the wetted area of a single droplet by the number of droplets in the horizontal array $\left(\mathrm{N}_{\mathrm{d}}\right)$.

To rigorously determine the motion of the droplet as it impinges on the dry tablet surface one would have to solve the mass and linear momentum balance equations, which is computationally demanding. In our work, we adopted a simplified approach based on the mechanical energy balance equation to develop a model that can quickly and accurately estimate the droplet spreading. The integral mechanical energy balance equation, which is derived from the generalized transport theorem (see Appendix), reads:

$$
\begin{aligned}
& \frac{\mathrm{d}}{\mathrm{dt}}\left[\int_{\mathcal{R}_{1}}\left(\frac{1}{2} \rho_{1} \mathbf{u}_{1} \cdot \mathbf{u}_{1}+\phi_{1}\right) \mathrm{d} \mathbf{x}+\int_{\mathcal{S}_{12}}\left(\frac{1}{2} \rho_{12} \mathbf{u}_{12} \cdot \mathbf{u}_{12}+\phi_{12}\right) \mathrm{d} \mathbf{s}+\int_{\mathcal{S}_{13}}\left(\frac{1}{2} \rho_{13} \mathbf{u}_{13} \cdot \mathbf{u}_{13}+\phi_{13}\right) \mathrm{d} \mathbf{s}\right. \\
& \left.\quad+\gamma_{12}\left(\mathrm{~A}_{12}-\mathrm{A}_{13} \cos \vartheta\right)\right]=\int_{\mathcal{S}_{12}} \mathbf{n}_{21} \cdot \sigma_{2} \cdot \mathbf{u}_{12} \mathrm{~d} \mathbf{s}+\int_{\mathcal{S}_{13}} \mathbf{n}_{31} \cdot \sigma_{3} \cdot \mathbf{u}_{13} \mathrm{~d} \mathbf{s}+\int_{\mathcal{R}_{1}} \tau_{1}: \partial_{\mathbf{x}} \mathbf{u}_{1} \mathrm{~d} \mathbf{x}
\end{aligned}
$$

where $\mathcal{S}_{12}$ is the interface between the liquid coating (phase 1) and the gas (phase 2) and $\mathcal{S}_{13}$ is the interface between the liquid and the solid (tablet surface; phase 3). The interfaces are shown in Figure 3. The three-dimensional spatial region bounded by the dividing surfaces $\mathcal{S}_{12}$ and $\mathcal{S}_{13}$ is denoted by $\mathcal{R}_{1}$. Moreover, $\mathbf{n}_{\mathrm{rs}}$ denotes the unit vector normal to $\mathcal{S}_{\mathrm{rs}}$ pointing from phase $\mathrm{r}$ into phase $\mathrm{s}$ and $\mathrm{A}_{\mathrm{rs}}$ denotes the area of $\mathcal{S}_{\mathrm{rs}}$. Here, $\rho_{\mathrm{r}}, \phi_{\mathrm{r}}$ and $\mathbf{u}_{\mathrm{r}}$ are the fluid density, the gravitational potential energy (per unit volume) and the fluid velocity for phase $r$, respectively, whereas $\rho_{\mathrm{rs}}, \phi_{\mathrm{rs}}$ and $\mathbf{u}_{\mathrm{rs}}$ are the fluid density, the gravitational potential energy (per unit surface) and the fluid velocity over the dividing surface $\mathcal{S}_{\mathrm{rs}}$, respectively. The stress tensor of phase $\mathrm{r}$ is denoted by $\sigma_{\mathrm{r}}$ and its deviatoric part by $\tau_{\mathrm{r}}$, whereas $\gamma_{12}$ is the surface tension on the surface $\mathcal{S}_{12}$ and $\vartheta$ is the dynamic contact angle of the three phases.

The left-hand side of Equation (1) features the rate of change of the total energy of the droplet, which comprises kinetic energy, potential energy of the gravitational field and surface energy, while the right-hand side features the rate of work performed by phases 2 and 3 (surrounding gas and solid tablet in our case) on the coating and the rate of conversion of mechanical energy into internal energy due to the action of the viscous stress within the liquid. We can write Equation (1) more concisely as:

$$
\frac{\mathrm{dE}_{\mathrm{K}}}{\mathrm{dt}}+\frac{\mathrm{dE}_{\mathrm{G}}}{\mathrm{dt}}+\frac{\mathrm{dE}_{\mathrm{S}}}{\mathrm{dt}}=\mathrm{W}_{\mathrm{S}}-\Phi
$$

where $\mathrm{E}_{\mathrm{K}}, \mathrm{E}_{\mathrm{G}}$ and $\mathrm{E}_{\mathrm{S}}$ are the kinetic, gravitational and surface energies of the droplet, respectively; $\Phi$ (which is positive) is the rate of viscous dissipation of the droplet kinetic energy and $\mathrm{W}_{\mathrm{S}}$ denotes the rate of work done by the droplet surroundings. 


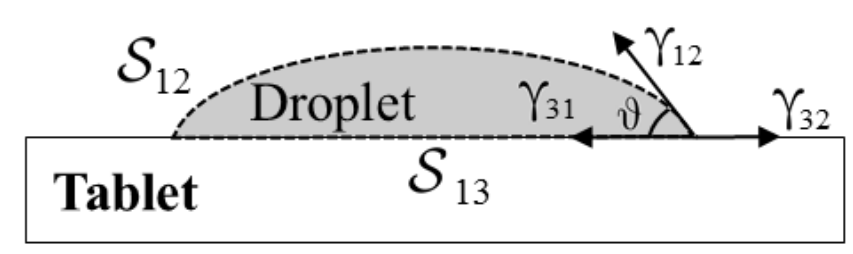

Figure 3: Spherical cap droplet on a tablet surface. $\vartheta$ is the dynamic contact angle and $\mathcal{S}_{\mathrm{rs}}$ and $\gamma_{\mathrm{rs}}$ are the interface and the interfacial tension between phase $\mathrm{r}$ and $\mathrm{s}$, respectively.

In the case of a single droplet impingement on a rigid substrate, the rate of work done by the surroundings can be neglected. Indeed, Equation (2) - without the term $\mathrm{W}_{\mathrm{S}}$ - has been repeatedly reported in the literature [17, 10]. However, to the best of our knowledge no rigorous derivation has been offered. We present our derivation of the mechanical energy balance equation for a droplet impinging on a solid substrate in the Appendix of this article.

To estimate the rate of change of the droplet gravitational potential energy $\left(\mathrm{dE}_{\mathrm{G}} / \mathrm{dt}\right)$ one can neglect the gravitational potential energy per unit surface $\left(\phi_{\mathrm{rs}}\right)$ over the liquid-air $\left(\mathcal{S}_{12}\right)$ and liquid-tablet $\left(\mathcal{S}_{13}\right)$ dividing surfaces. Thus, considering significant only the gravitational potential energy of the coating droplet per unit volume, $\phi_{1}$, we write:

$$
\frac{\mathrm{dE}_{\mathrm{G}}}{\mathrm{dt}} \approx \frac{\mathrm{d}}{\mathrm{dt}} \int_{\mathcal{R}_{1}} \phi_{1} \mathrm{dx}=\frac{\mathrm{d}}{\mathrm{dt}}\left(\frac{\pi}{6} \mathrm{D}_{0}^{3} \rho_{1} \mathbf{g} \mathrm{h}_{\mathrm{m}}\right)
$$

where $\mathbf{g}$ is the gravitational field, $\mathrm{D}_{0}$ is the droplet diameter before impact and $\mathrm{h}_{\mathrm{m}}$ is the distance from the tablet surface to the droplet center of mass. The gravitational potential energy of an impinging droplet is usually neglected [10] if the droplet Bond number is small $\left(\mathrm{Bo}_{0} \equiv \mathrm{\rho}_{1} \mathrm{~g} \mathrm{D}_{0} / \gamma_{12}<<1\right)$.

The rate of change of the droplet surface energy appears on the left-hand side of Equation (1):

$$
\frac{\mathrm{dE}}{\mathrm{dt}}=\frac{\mathrm{d}}{\mathrm{dt}}\left[\gamma_{12}\left(\mathrm{~A}_{12}-\mathrm{A}_{13} \cos \vartheta\right)\right]
$$

where $\mathrm{A}_{12}$ and $\mathrm{A}_{13}$ are the areas of the liquid-gas and liquid-solid interfaces, respectively. In this work, we assumed that the surface tension $\left(\gamma_{12}\right)$ is constant and uniform (that is, independent of position). In Equations (1) and (4), $\vartheta$ is the dynamic contact angle which we assumed to be approximately equal to the equilibrium contact angle (Fig. 3).

To calculate the kinetic energy of the droplet and the rate of viscous dissipation of kinetic energy, we need to know the velocity field $\mathbf{u}_{1}$ inside the control volume (coating droplet of volume $\mathrm{V}$ ). To obtain this, we would have to solve the mass and linear momentum balance equations; we decided not to do so, favoring a less computationally demanding modeling approach. The consequence of this choice is that now to proceed we need to assume a functional form for the velocity field.

The form of the velocity field can vary considerably inside the droplet. For the impact conditions of interest in our work, we can assume that the droplet presents two regions in which the velocity field is substantially different; the first region spans almost the entire droplet and in it the flow can be approximated as inviscid, while the second region is a boundary layer of thickness $\delta$ near the tablet surface $\left(\mathcal{S}_{13}\right)$ where viscous dissipation is significant.

Batchelor [25] estimated the boundary layer thickness for this flow, showing that:

$$
\frac{\delta}{\mathrm{h}_{\mathrm{m}}} \sim\left(\frac{\mu_{1}}{\rho_{1} \mathrm{U}_{\mathrm{m}} \mathrm{h}_{\mathrm{m}}}\right)^{1 / 2} \sim\left(\frac{\mu_{1}}{\rho_{1} \mathrm{U}_{0} \mathrm{D}_{0}}\right)^{1 / 2} \sim \frac{1}{\sqrt{\mathrm{Re}}}
$$

where $R e \equiv \rho_{1} U_{0} D_{0} / \mu_{1}$ is the impact Reynolds number, $U_{m}$ is the velocity of the droplet center of mass $\left(\mathrm{dh}_{\mathrm{m}} / \mathrm{dt}\right)$ and $\mu_{1}$ is the liquid coating viscosity. In our coating droplet simulations, $150<\operatorname{Re}<600$ and so $\delta / h_{\mathrm{m}} \sim 0.01$; the boundary layer is thus much thinner than the inviscid-flow bulk region. 
Based on the above, the velocity field in the inviscid-flow region of the droplet can be used to calculate the total kinetic energy of the droplet, since the boundary layer, being thin, does not contribute significantly to this quantity. In contrast, the velocity field in the boundary layer should be used to calculate the rate of viscous dissipation of the droplet kinetic energy, since the contribution of the inviscid-flow region to this quantity is negligible.

To calculate the kinetic energy, we assumed that the flow field in the bulk of the droplet is axisymmetric near a stagnation point $(\mathrm{O})$, which is located on the tablet surface where the droplet impinges (Fig. 4). This type of flow is known as "stagnation point flow" [25]. The motion of the droplet is then described in terms of a stream function $\psi(\mathrm{r}, \mathrm{z}, \mathrm{t})$, where $\mathrm{r}$ and $\mathrm{z}$ are the radial and vertical cylindrical coordinates, respectively. In particular, the radial $\left(\mathrm{u}_{1 \mathrm{r}}\right)$ and vertical $\left(\mathrm{u}_{1 \mathrm{z}}\right)$ velocity components are given by:

$$
\mathrm{u}_{1 \mathrm{r}}=-(1 / \mathrm{r}) \partial_{\mathrm{z}} \psi ; \quad \mathrm{u}_{1 \mathrm{z}}=(1 / \mathrm{r}) \partial_{\mathrm{r}} \psi
$$

while the angular velocity component is zero. The stream function for irrotational flow near a stagnation point is well documented in the literature and is given by $\psi=(\mathrm{k} / 2) \mathrm{zr}^{2}$, where $\mathrm{k}$ is a constant [25]. Substituting this function in Equation (6) gives:

$$
\mathrm{u}_{1 \mathrm{r}}=-\frac{1}{2} \mathrm{kr} \quad ; \quad \mathrm{u}_{1 \mathrm{z}}=\mathrm{kz}
$$

For an impinging droplet, the value of the parameter $\mathrm{k}$ can be calculated by considering the relations (7) at the droplet center of mass:

$$
\mathrm{u}_{1 \mathrm{r}}\left(0, \mathrm{~h}_{\mathrm{m}}, \mathrm{t}\right)=0 ; \mathrm{u}_{1 \mathrm{z}}\left(0, \mathrm{~h}_{\mathrm{m}}, \mathrm{t}\right)=\frac{\mathrm{dh}_{\mathrm{m}}}{\mathrm{dt}}=\mathrm{kh}_{\mathrm{m}} \Rightarrow \mathrm{k}=\frac{\mathrm{U}_{\mathrm{m}}}{\mathrm{h}_{\mathrm{m}}}
$$

Substituting k back into Equation (7) yields:

$$
\begin{gathered}
\mathrm{u}_{1 \mathrm{r}}=-\frac{1}{2} \frac{\mathrm{r}}{\mathrm{h}_{\mathrm{m}}} \mathrm{U}_{\mathrm{m}} \\
\mathrm{u}_{1 \mathrm{z}}=\frac{\mathrm{z}}{\mathrm{h}_{\mathrm{m}}} \mathrm{U}_{\mathrm{m}}
\end{gathered}
$$

The above equations satisfy the continuity equation for an incompressible fluid. Expressing the kinetic energy $\left(\mathrm{E}_{\mathrm{K}}\right)$ of the droplet in terms of the velocity components for the "stagnation point flow" (Equations 9,10) and neglecting the kinetic energy associated with the dividing surfaces $\mathcal{S}_{12}$ and $\mathcal{S}_{13}$ yields:

$$
\mathrm{E}_{\mathrm{K}} \approx \frac{1}{2} \rho_{1} \int_{\mathcal{R}_{1}} \mathbf{u}_{1} \cdot \mathbf{u}_{1} \mathrm{~d} \mathbf{x}=\frac{1}{2} \rho_{1} \int_{\mathcal{R}_{1}}\left(\mathrm{u}_{1 \mathrm{r}}^{2}+\mathrm{u}_{1 \mathrm{z}}^{2}\right) \mathrm{d} \mathbf{x} \approx \frac{1}{2} \rho_{1} \int_{\mathcal{R}_{1}}\left[\left(-\frac{1}{2} \frac{\mathrm{r}}{\mathrm{h}_{\mathrm{m}}} \mathrm{U}_{\mathrm{m}}\right)^{2}+\left(\frac{\mathrm{z}}{\mathrm{h}_{\mathrm{m}}} \mathrm{U}_{\mathrm{m}}\right)^{2}\right] \mathrm{d} \mathbf{x}
$$

Here we integrated over the entire region $\mathcal{R}_{1}$ occupied by the impinging droplet instead of restricting the integration to the inviscid-flow region. This does not affect the result significantly, since in our simulations the boundary layer (where viscous dissipation is not negligible) is thin. To calculate the integral above, we assumed that the droplet has a spherical cap shape (Fig. 2b), so that the following geometrical relations hold [14]:

$$
\mathrm{R}_{\mathrm{d}}^{2}=\frac{\mathrm{D}_{0}^{2}}{3}\left(\frac{\mathrm{D}_{0}}{\mathrm{~h}_{\max }}-\frac{\mathrm{h}_{\max }^{2}}{\mathrm{D}_{0}^{2}}\right) ; \mathrm{h}_{\mathrm{m}}=\frac{1}{6}\left(2 \mathrm{~h}_{\max }+\frac{\mathrm{h}_{\max }^{4}}{\mathrm{D}_{0}^{3}}\right)
$$

where $R_{d}$ and $h_{\max }$ are the time-dependent droplet wetted area radius and maximum droplet height, respectively. Integrating Equation (11) over the volume of a spherical cap described by Equation (12), and taking the time derivative, yields:

$$
\frac{d E_{K}}{d t}=\frac{d}{d t}\left[\frac{\rho_{1} \pi U_{m}^{2}}{2 h_{m}^{2}}\left(\frac{R_{d}^{4} h_{\max }}{16}+\frac{13 R_{d}^{2} h_{\max }^{3}}{72}+\frac{h_{\max }^{5}}{10}\right)\right]
$$




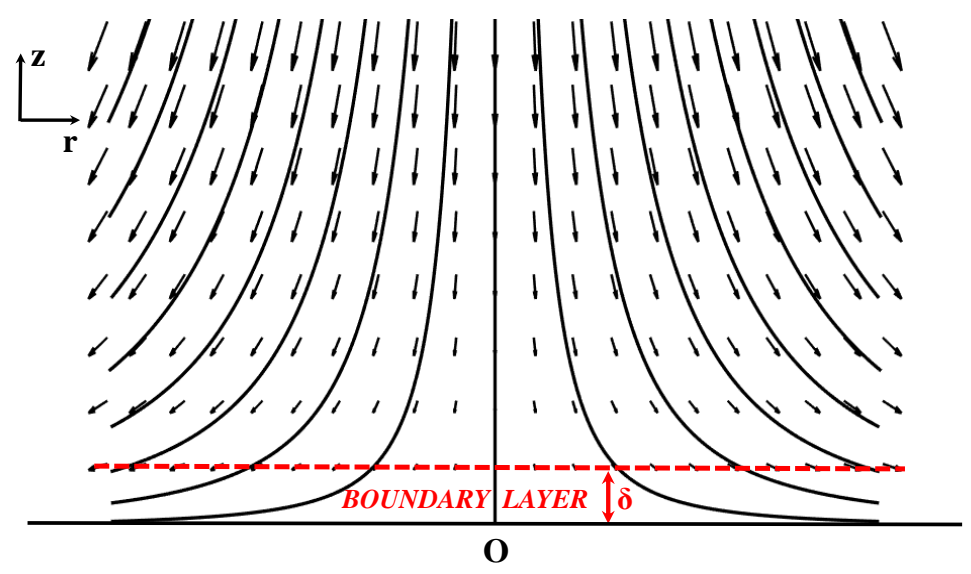

Figure 4: Axisymmetric stagnation point flow. The lines denote the flow streamlines.

Viscous dissipation during droplet impingement occurs in the boundary layer and the bulk of the droplet. Since the boundary layer is thin, one can calculate the rate of viscous dissipation in the latter region by integrating over $\mathcal{R}_{1}$ as follows:

$$
\begin{gathered}
\Phi_{\mathrm{b}}=-\int_{\mathcal{R}_{1}} \tau_{1}: \partial_{\mathbf{x}} \mathbf{u}_{1} \mathrm{~d} \mathbf{x}=2 \mu_{1} \int_{\mathcal{R}_{1}}\left[\left(\partial_{\mathrm{r}} \mathrm{u}_{1 \mathrm{r}}\right)^{2}+\left(\mathrm{u}_{1 \mathrm{r}} / \mathrm{r}\right)^{2}+\left(\partial_{\mathrm{z}} \mathrm{u}_{1 \mathrm{z}}\right)^{2}+\frac{1}{2}\left(\partial_{\mathrm{z}} \mathrm{u}_{1 \mathrm{r}}+\partial_{\mathrm{r}} \mathrm{u}_{1 \mathrm{z}}\right)^{2}\right] \mathrm{d} \mathbf{x} \\
=3 \mu_{1} \int_{\mathcal{R}_{1}}\left(\frac{1}{\mathrm{~h}_{\mathrm{m}}} \mathrm{U}_{\mathrm{m}}\right)^{2} \mathrm{~d} \mathbf{x}=\frac{\mu_{1} \pi \mathrm{U}_{\mathrm{m}}^{2}}{2} \frac{\mathrm{h}_{\max }}{\mathrm{h}_{\mathrm{m}}^{2}}\left(3 \mathrm{R}_{\mathrm{d}}^{2}+\mathrm{h}_{\max }^{2}\right)
\end{gathered}
$$

In the boundary layer viscous dissipation is significant [15]. Since in this region we do not have a functional form for the velocity field $\mathbf{u}_{1}$, we cannot solve the volume integral rigorously as we did in Equations (11) and (14); however, we can estimate the value of the rate of viscous dissipation in the boundary layer $\Phi_{\widehat{\delta}}$ using scaling analysis. In the Appendix of this article, we show that the $\Phi_{\delta}$ estimate can be obtained as follows:

$$
\Phi_{\delta}=-\int_{\mathcal{R}_{\tilde{\delta}}} \tau_{1}: \partial_{\mathbf{x}} \mathbf{u}_{1} \mathrm{~d} \mathbf{x} \sim \frac{\mu_{1}}{\delta^{2}} \int_{\mathcal{R}_{\hat{\delta}}} \mathrm{u}_{1 \mathrm{r}}^{2} \mathrm{~d} \mathbf{x} \sim \frac{\mu_{1}}{\delta} \int_{\mathcal{S}_{13}} \mathrm{u}_{1 \mathrm{r}}^{2} \mathrm{~d} \mathbf{s} \sim \frac{\mu_{1}}{\mathrm{~h}_{\mathrm{m}}}\left(\frac{\rho_{1} \mathrm{U}_{\mathrm{m}} \mathrm{h}_{\mathrm{m}}}{\mu_{1}}\right)^{1 / 2} \int_{\mathcal{S}_{13}}\left(-\frac{1}{2} \frac{\mathrm{r}}{\mathrm{h}_{\mathrm{m}}} \mathrm{U}_{\mathrm{m}}\right)^{2} \mathrm{~d} \mathbf{s}
$$

where $\mathcal{R}_{\widehat{\delta}}$ is the boundary layer region and $\mathcal{S}_{13}$ is the droplet-tablet dividing surface that corresponds to the wetted area. This expression estimates correctly the order of magnitude of $\Phi_{\delta}$; however, to obtain a more accurate result, we introduced an empirical dissipation factor, denoted as $\mathrm{F}_{\mathrm{d}}$. Its derivation is discussed later in this section. With the introduction of the dissipation factor, the expression yields:

$$
\Phi_{\delta} \approx \frac{F_{d} \mu_{1} U_{m}^{2}}{4 h_{m}^{3}}\left(\frac{\rho_{1} U_{m} h_{m}}{\mu_{1}}\right)^{1 / 2} \int_{0}^{2 \pi} \int_{0}^{R_{d}} r^{3} \mathrm{dr} d \varphi=\frac{F_{d} \mu_{1} U_{m}^{2} \pi R_{d}^{4}}{8 h_{m}^{3}}\left(\frac{\rho_{1} U_{m} h_{m}}{\mu_{1}}\right)^{1 / 2}
$$

Because the velocity field inside the bulk of the droplet is essentially inviscid, estimating the rate of viscous dissipation only from Equation (14) would grossly underestimate the value of the viscous dissipation rate. Indeed, since in our simulations we have $\mathrm{h}_{\max } \sim \mathrm{R}_{\mathrm{d}}$, using scaling to compare $\Phi_{\mathrm{b}}$ and $\Phi_{\delta}$ yields: $\Phi_{\mathrm{b}} / \Phi_{\delta} \sim \delta / \mathrm{h}_{\mathrm{m}}<<1$. Thus, we considered $\Phi \approx \Phi_{\delta}$ in the mechanical energy balance Equation (2).

To solve the mechanical energy balance (Equation 2), we substituted the expressions for the gravitational, surface and kinetic energies, as well as the rate of viscous dissipation given by Equations (3), (4), (13) and (15), respectively.

Nevertheless, to render the equation solvable, an expression for the factor $F_{d}$ is needed. Empirical expressions for the dissipation factor have been derived by Bechtel et al. [14] and Kim and Chun [15], who fitted their experimental data for water droplets impact to obtain $\mathrm{F}_{\mathrm{d}}=5.3 \mathrm{Oh}^{-1 / 2}$ and $\mathrm{F}_{\mathrm{d}}=\sqrt{\pi} \mathrm{Oh}^{-1 / 2}$, respectively. Here Oh denotes the Ohnesorge number $\left(\mathrm{Oh} \equiv \mu / \sqrt{\mathrm{\rho \gamma} \mathrm{D}_{0}}\right)$. 
Being interested in pharmaceutical coatings, we fitted $\mathrm{F}_{\mathrm{d}}$ to match the experimental data of Bolledulla et al. [10] for the spreading of various coating droplets impinging on tablets with different Ohnesorge $(\mathrm{Oh})$ number values. We used gPROMS Modelbuilder [26] to estimate the values of $\mathrm{F}_{\mathrm{d}}$ which can be introduced in our model to approximate the wetted area radius after impact of several OpadryII White coating droplets (with ranging solid concentrations) and a glycerol/water solution droplet. In all case studies chosen for the fitting we have Re $\sim 100$. By plotting the values of $F_{d}$ against the corresponding ones of the Ohnesorge number (Fig. 5) and by using a regression, we derived the expression:

$$
\mathrm{F}_{\mathrm{d}}=1.868 \mathrm{Oh}^{-0.431}
$$

Equation (17) holds for the Opadry coating droplets used in the experiments with which we validated the results of our model (Section 3.1). Note, that in the validation section the model is predictive, and not fitted, since we used it to analyze case studies different from those used in the derivation of Equation (17). The dissipation factor proposed in this work could also be used to estimate viscous dissipation of various coating liquids for moderate droplet Reynolds and Ohnesorge numbers $(\operatorname{Re} \sim 100, \mathrm{Oh} \sim 0.1)$.

The initial conditions required to solve the mechanical energy balance equation are:

$$
\left.\frac{\mathrm{dh}_{\mathrm{m}}}{\mathrm{dt}}\right|_{\mathrm{t}=0}=-\mathrm{U}_{0} ;\left.\mathrm{h}_{\max }\right|_{\mathrm{t}=0}=\mathrm{D}_{0}
$$

We wrote the mechanical energy balance equation in terms of just one independent variable, which we chose to be $h_{m}$. To do so, we substituted the expressions for the droplet potential (gravitational + surface) energy, kinetic energy and rate of viscous dissipation, given by Equations (3), (4), (11) and (15), respectively, into Equation (2) and then used the geometrical relations (12) to eliminate the variables $R_{d}$ and $h_{\max }$. Since all the droplets of the first array behave independently and identically, we calculated the equivalent radius $\left(R_{\text {eq }}\right)$ of the wetted area at any given time $t<t_{i}$ as:

$$
\pi \mathrm{R}_{\text {eq }}^{2}=\mathrm{N}_{\mathrm{d}}\left(\pi \mathrm{R}_{\mathrm{d}}^{2}\right) \quad ; \quad \mathrm{N}_{\mathrm{d}}=\left(\dot{\mathcal{Q}}_{\mathrm{t}} / \mathrm{V} \rho_{1}\right) \mathrm{t}_{\mathrm{i}}
$$

where $\mathrm{N}_{\mathrm{d}}$ is the number of droplets in each array, which depends on the spray mass flow rate applied to the tablet $\left(\dot{\mathcal{Q}}_{t}\right)$ and on the characteristic time $t_{\mathrm{i}}$. The mass flow rate of coating applied on a single tablet can be calculated from the overall spray mass flow rate $(\dot{\mathcal{Q}})$, which is a process parameter, if the ratio of tablet-bed to single-tablet surface area $\left(\mathrm{L} \equiv \mathrm{A}_{\text {bed }} / \mathrm{A}_{\mathrm{t}}\right)$ is known, since $\dot{\mathcal{Q}}_{\mathrm{t}}=\dot{\mathcal{Q}} / \mathrm{L}$.

To summarize, the model presented in this section describes droplet spreading until the second array of droplets impinges on top of the first at $t=t_{i}$. The subsequent droplets of the spray will impact on the initial wetted surface leading to further spreading and thickening of the coating film. Successive droplet impacts on the wetted surface are discussed in the next section.

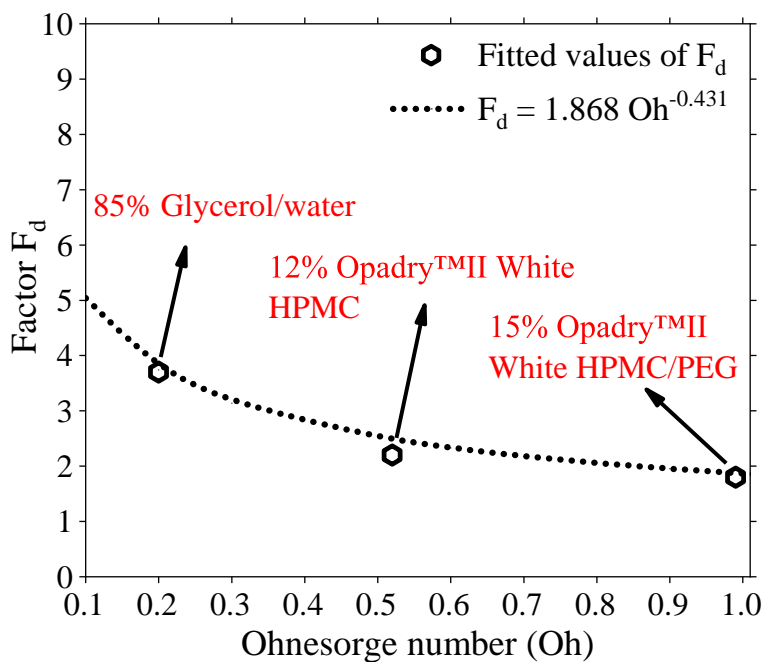

Figure 5: $\mathrm{F}_{\mathrm{d}}$ factor estimation. The experimental data are from Bolleddula et al. (2010). 
(a)

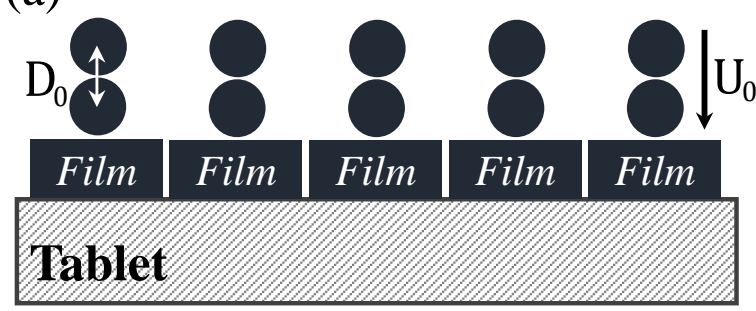

(b)

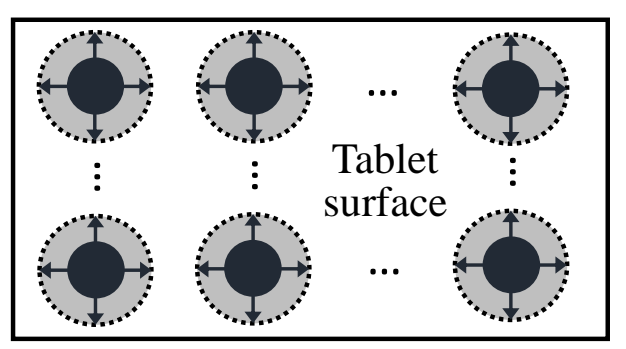

Figure 6: Successive droplet impact on the tablet. a) Side and b) top view of the tablet.

\subsection{Droplet impingement on wetted surface}

Droplets of the spray which follow directly behind the first array will impinge on the wetted area (calculated in Section 2.1) and will contribute to its spreading. We assumed that at this stage the wetted area is made up of disconnected identical cylindrical films (Fig. 6) that expand axisymmetrically. Over a time interval dt, the impingement of a droplet on the surface of a film increases its volume by $\mathrm{dV}_{\mathrm{f}}=\left(\dot{\mathcal{Q}}_{\mathrm{i}} / \rho_{1}\right) \mathrm{dt}$, where $\dot{\mathcal{Q}}_{\mathrm{i}}$ is the droplet mass flow rate applied to each disconnected film $\left(\dot{\mathcal{Q}}_{\mathrm{i}}=\dot{\mathcal{Q}}_{\mathrm{t}} / \mathrm{N}_{\mathrm{d}}\right)$. Thus, the volume of each film at any given time $t+d t>t_{i}$ can be calculated by $V_{f}(t+d t)=V_{f}(t)+d V_{f}$. The coating application is assumed to be completed when the wetted area reaches the area of the tablet surface that is facing the spray $\left(A_{t}\right)$.

Similarly to the single droplet case (Section 2.1), we assumed that the spreading of each disconnected film of volume $V_{\mathrm{f}}$ is governed by the mechanical energy balance equation:

$$
\frac{\mathrm{dE}_{\mathrm{Kf}}}{\mathrm{dt}}+\frac{\mathrm{dE}_{\mathrm{Gf}}}{\mathrm{dt}}+\frac{\mathrm{dE}_{\mathrm{Sf}}}{\mathrm{dt}}=\mathrm{W}_{\mathrm{Sf}}-\Phi_{\mathrm{f}}
$$

where $\mathrm{E}_{\mathrm{Kf}}, \mathrm{E}_{\mathrm{Gf}}$ and $\mathrm{E}_{\mathrm{Sf}}$ are the kinetic, gravitational and surface energies of each film, respectively, $\Phi_{\mathrm{f}}$ is the rate of viscous dissipation of the kinetic energy of the film and $\mathrm{W}_{\mathrm{Sf}}$ denotes the rate of work done on the film surface by the surroundings. The key difference is that the first term on the right-hand side, which denotes the rate of work done by the surroundings on the surface of the film, cannot be neglected. The part of this work that in particular needs to be accounted for is that carried out by the impinging droplets.

Similarly to the single droplet case, we calculated the rate of change of the gravitational and surface energies of each cylindrical film:

$$
\begin{gathered}
\frac{d E_{G f}}{d t}=\frac{d}{d t}\left(V_{f} \rho_{1} g H_{m}\right) \\
\frac{d E_{S f}}{d t}=\frac{d}{d t}\left[2 \gamma_{12} \pi R_{f} H_{f}+\gamma_{12} \pi R_{f}^{2}(1-\cos \vartheta)\right]
\end{gathered}
$$

where $H_{f}, H_{m}$ and $R_{f}$ represent the film thickness, center of mass height and wetted area radius, respectively. Here, the coating film center of mass height is equal to half of the film thickness $\left(\mathrm{H}_{\mathrm{m}}=\mathrm{H}_{\mathrm{f}} / 2\right)$.

To calculate the kinetic energy rate of change and the rate of viscous dissipation terms in Equation (20), one needs to assume a functional form for the velocity field inside the spreading films. We followed the approach of Madejski [27], who proposed the following shear flow velocity field (Fig. 7):

$$
\mathrm{u}_{1 \mathrm{r}}=\mathrm{Crz} ; \mathrm{u}_{1 \mathrm{z}}=-\mathrm{Cz}^{2}
$$

where $\mathrm{r}$ and $\mathrm{z}$ are the radial and vertical cylindrical coordinates. By assuming that the wetted area expansion $\left(d R_{f} / d t\right)$ is equal to the average radial velocity at $r=R_{f}$, we can write:

$$
\frac{\mathrm{dR}_{\mathrm{f}}}{\mathrm{dt}}=\frac{1}{\mathrm{H}_{\mathrm{f}}} \int_{0}^{\mathrm{H}_{\mathrm{f}}} \mathrm{u}_{1 \mathrm{r}}\left(\mathrm{R}_{\mathrm{f}}, \mathrm{z}\right) \mathrm{dz}=\frac{\mathrm{CR}_{\mathrm{f}}}{\mathrm{H}_{\mathrm{f}}} \int_{0}^{\mathrm{H}_{\mathrm{f}}} \mathrm{zdz} \Rightarrow \mathrm{C}=\frac{2}{\mathrm{R}_{\mathrm{f}} \mathrm{H}_{\mathrm{f}}} \frac{\mathrm{dR}_{\mathrm{f}}}{\mathrm{dt}}
$$




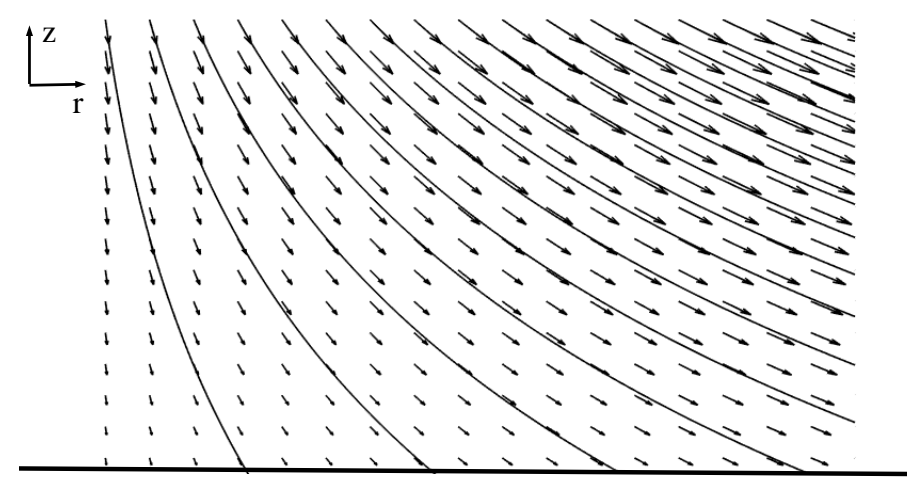

Figure 7: Shear flow velocity field. The dotted lines denote the streamlines.

Substituting C into Equation (23) gives:

$$
\begin{gathered}
\mathrm{u}_{1 \mathrm{r}}=\frac{2 \mathrm{rz}}{\mathrm{R}_{\mathrm{f}} \mathrm{H}_{\mathrm{f}}} \frac{\mathrm{dR}_{\mathrm{f}}}{\mathrm{dt}} \\
\mathrm{u}_{1 \mathrm{z}}=-\frac{2 \mathrm{z}^{2}}{\mathrm{R}_{\mathrm{f}} \mathrm{H}_{\mathrm{f}}} \frac{\mathrm{dR}_{\mathrm{f}}}{\mathrm{dt}}
\end{gathered}
$$

Using scaling arguments we prove in the Appendix of this article that the above velocity components are considerably smaller than the droplet impact velocity $\mathrm{U}_{0}$. Thus, the velocity field inside the film does not vary considerably during droplet impingement on its surface and therefore no boundary layer exists. We used the velocity components (Equations 25,26) to calculate the rate of change of kinetic energy $\left(\mathrm{dE}_{\mathrm{Kf}} / \mathrm{dt}\right)$ and viscous dissipation loss rate $\left(\Phi_{\mathrm{f}}\right)$ of each cylindrical film of volume $\mathrm{V}_{\mathrm{f}}$ as follows:

$$
\begin{gathered}
\frac{\mathrm{dE} E_{\mathrm{Kf}}}{\mathrm{dt}}=\frac{\mathrm{d}}{\mathrm{dt}}\left(\frac{1}{2} \rho_{1} \int_{\mathcal{R}_{\mathrm{f}}} \mathbf{u}_{1} \cdot \mathbf{u}_{1} \mathrm{~d} \mathbf{x}\right)=\frac{1}{2} \rho_{1} \frac{\mathrm{d}}{\mathrm{dt}}\left[\int_{\mathcal{R}_{\mathrm{f}}}\left(\mathrm{u}_{1 \mathrm{r}}^{2}+\mathrm{u}_{1 \mathrm{z}}^{2}\right) \mathrm{d} \mathbf{x}\right] \\
\approx \frac{\mathrm{d}}{\mathrm{dt}}\left[\frac{\rho_{1} \pi \mathrm{H}_{\mathrm{f}}}{60}\left(6 \mathrm{H}_{\mathrm{f}}^{2}+5 \mathrm{R}_{\mathrm{f}}^{2}\right)\left(\frac{\mathrm{dR}}{\mathrm{dt}}\right)^{2}\right] \\
\Phi_{\mathrm{f}}=-\int_{\mathcal{R}_{\mathrm{f}}} \tau_{1}: \partial_{\mathrm{x}} \mathbf{u}_{1} \mathrm{~d} \mathbf{x}=2 \mu_{1} \int_{\mathcal{R}_{\mathrm{f}}}\left[\left(\partial_{\mathrm{r}} \mathrm{u}_{1 \mathrm{r}}\right)^{2}+\left(\mathrm{u}_{1 \mathrm{r}} / \mathrm{r}\right)^{2}+\left(\partial_{\mathrm{z}} \mathrm{u}_{1 \mathrm{z}}\right)^{2}+\frac{1}{2}\left(\partial_{\mathrm{z}} \mathrm{u}_{1 \mathrm{r}}+\partial_{\mathrm{r}} \mathrm{u}_{1 \mathrm{z}}\right)^{2}\right] \mathrm{d} \mathbf{x} \\
\approx \frac{2 \mu_{1} \pi}{\mathrm{H}_{\mathrm{f}}}\left(\mathrm{R}_{\mathrm{f}}^{2}+8 \mathrm{H}_{\mathrm{f}}^{2}\right)\left(\frac{\mathrm{dR}}{\mathrm{dt}}\right)^{2}
\end{gathered}
$$

In Equation (27), we neglected the kinetic energy associated with the liquid-air $\left(\mathcal{S}_{12}\right)$ and liquid-tablet $\left(\mathcal{S}_{13}\right)$ dividing surfaces and we integrated over the entire region $\mathcal{R}_{\mathrm{f}}$ occupied by each cylindrical film.

The rate of work done by the surroundings (phases 2 and 3) on the surface of each film is given by:

$$
\mathrm{W}_{\mathrm{Sf}}=\int_{\mathcal{S}_{12}} \mathbf{n}_{21} \cdot \sigma_{2} \cdot \mathbf{u}_{12} \mathrm{~d} \mathbf{s}+\int_{\mathcal{S}_{\mathrm{i}}} \mathbf{n}_{\mathrm{df}} \cdot \sigma_{1} \cdot \mathbf{u}_{1} \mathrm{~d} \mathbf{s}+\int_{\mathcal{S}_{13}} \mathbf{n}_{31} \cdot \sigma_{3} \cdot \mathbf{u}_{13} \mathrm{~d} \mathbf{s}
$$

where $\mathcal{S}_{\mathrm{i}}$ is the droplet-film dividing surface and $\mathbf{n}_{\mathrm{df}}$ is the unit vector normal to $\mathcal{S}_{\mathrm{i}}$ pointing from the droplet to the film. In Equation (29), we can neglect the first term on the right-hand side which denotes the rate of work done on the surface $\mathcal{S}_{12}$ by the surrounding gas; this is the same approximation used in Section 2.1, where we treated a single droplet impinging on a dry substrate. Since the fluid velocity at the tablet-film interface $\mathcal{S}_{13}$ is zero $\left(\mathbf{u}_{13}=0\right.$; no-slip boundary condition), the third term on the right-hand side is zero. The rate of work done by the impinging droplets on the surface of each cylindrical film can be repartitioned into two contributions:

$$
\mathrm{W}_{\mathrm{Sf}} \approx \int_{\mathcal{S}_{\mathrm{i}}} \mathbf{n}_{\mathrm{df}} \cdot \sigma_{1} \cdot \mathbf{u}_{1} \mathrm{~d} \mathbf{s}=\int_{\mathcal{S}_{\mathrm{i}}} \mathrm{p}_{1} \mathbf{n}_{\mathrm{df}} \cdot \mathbf{u}_{1} \mathrm{~d} \mathbf{s}+\int_{\mathcal{S}_{\mathrm{i}}} \mathbf{n}_{\mathrm{df}} \cdot \tau_{1} \cdot \mathbf{u}_{1} \mathrm{~d} \mathbf{s}
$$

where $\mathrm{p}_{1}$ is the pressure in the coating liquid. The two terms on the right-hand side represent the rates of work done by the pressure and viscous forces, respectively, on the surface of the film. 

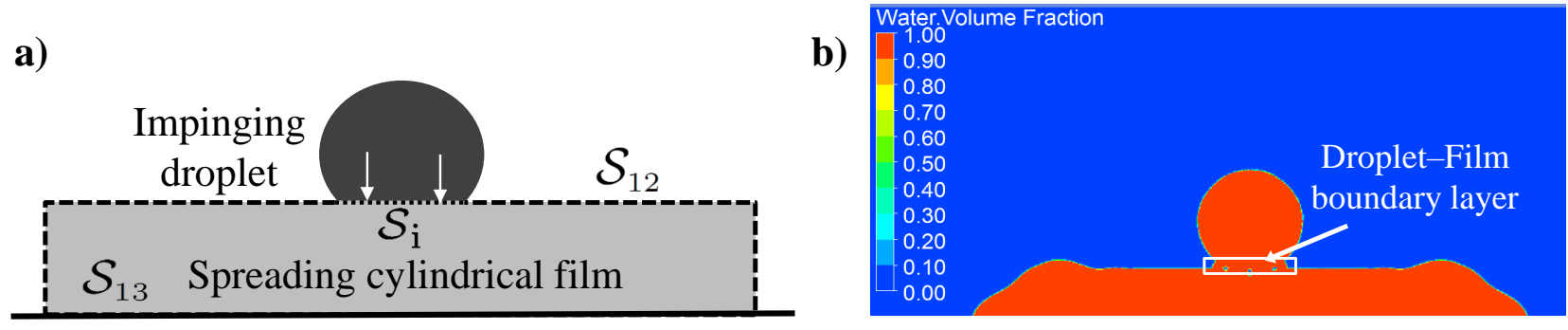

Figure 8: a) Droplet impact on a film, b) Volume-Of-Fluid CFD simulation of droplet-film impingement.

Let us first deal with the pressure term on the right-hand side of Equation (30). To calculate the work done by the pressure forces on $\mathcal{S}_{\mathrm{i}}$, one needs an expression for the pressure field. To derive it, we assumed that the region of the droplet near the interface (droplet-film boundary layer, Fig. 8.b) decelerates significantly upon impact (in this region the velocity field magnitude is far less than $\mathrm{U}_{0}$ ). In fact, using scaling arguments, we can write:

$$
\frac{\mathrm{u}_{1 \mathrm{z}}}{\mathrm{U}_{0}} \sim \frac{\mathrm{CH}_{\mathrm{f}}^{2}}{\mathrm{U}_{0}} \sim \frac{\mathrm{H}_{\mathrm{f}}}{\mathrm{R}_{\mathrm{f}} \mathrm{U}_{0}} \frac{\mathrm{dR}_{\mathrm{f}}}{\mathrm{dt}} \sim\left(\frac{\mathrm{H}_{\mathrm{f}}}{\mathrm{R}_{\mathrm{f}}}\right)^{2} \sim\left(\frac{\mathrm{D}_{0}}{\mathrm{R}_{\mathrm{f}}}\right)^{2}<<1
$$

In the last passage (Equation 31), we have assumed that the thickness of the coating film has the same order of magnitude as $D_{0}$ which is true in our simulations, and that $d R_{f} / d t \sim\left(H_{f} / R_{f}\right) U_{0}$ which is shown to be true using scaling arguments in the Appendix of this article. Notice that at the very beginning of the spray application, $\mathrm{R}_{\mathrm{f}} \sim \mathrm{H}_{\mathrm{f}}$ and so the velocity in the droplet-film boundary layer and the droplet impact velocity have the same order of magnitude. Note that the time interval over which this condition holds is very short compared to that over which the tablet is sprayed $(\sim 0.1 \mathrm{~s}$, [29]). For most of the time, $\mathrm{H}_{\mathrm{f}} / \mathrm{R}_{\mathrm{f}}<<1$ and consequently the droplet impact velocity is significantly larger than the vertical velocity in the film. This has been also confirmed numerically (refer to the CFD simulations discussed in the Appendix).

We conclude that in the droplet-film boundary layer the kinetic energy of the fluid turns almost entirely into pressure energy, so that in this region $\mathrm{p}_{1} \approx(1 / 2) \rho_{1} \mathrm{U}_{0}^{2}$. We can thus write:

$$
\int_{\mathcal{S}_{\mathrm{i}}} \mathrm{p}_{1} \mathbf{n}_{\mathrm{df}} \cdot \mathbf{u}_{1} \mathrm{~d} \mathbf{s} \sim(1 / 2) \rho_{1} \mathrm{U}_{0}^{2} \int_{\mathcal{S}_{\mathrm{i}}} \mathbf{n}_{\mathrm{df}} \cdot \mathbf{u}_{1} \mathrm{~d} \mathbf{s}=(1 / 2) \mathrm{U}_{0}^{2} \dot{\mathrm{m}}(\mathrm{t})
$$

where $\dot{\mathrm{m}}$ is the droplet mass entering in/merging with the film per unit time. This quantity is time-dependent, but its order of magnitude is given by the ratio between the droplet mass $M_{D}$ and the time $t_{i}$ required by the droplet to fully merge with the film. Based on the above we obtain:

$$
\int_{\mathcal{S}_{\mathrm{i}}} \mathrm{p}_{1} \mathbf{n}_{\mathrm{df}} \cdot \mathbf{u}_{1} \mathrm{~d} \mathbf{s} \equiv \mathrm{W}_{\mathrm{p}} \sim(1 / 2) \mathrm{U}_{0}^{2} \mathrm{M}_{\mathrm{D}} / \mathrm{t}_{\mathrm{i}}=(1 / 2) \mathrm{U}_{0}^{2} \dot{\mathcal{Q}}_{\mathrm{i}}
$$

where $\dot{\mathcal{Q}}_{\text {i }}$ denotes the droplet mass flow rate applied to each disconnected cylindrical film; this is equal to the ratio $\dot{\mathcal{Q}}_{\mathrm{t}} / \mathrm{N}_{\mathrm{d}}$, in which the values of both quantities can be regarded as known (refer to the previous section).

As before, to calculate the rate of work done by viscous forces on each film $\left(\mathrm{W}_{\mathrm{V}}\right)$, we considered the no-slip boundary condition at the tablet film interface, and we neglected the rate of work done on $\mathcal{S}_{12}$ and we considered significant the rate of work done by the viscous forces only inside the droplet region that decelerates upon impact (the droplet-film boundary layer). Pasandideh-Fard et al. [28] report that the droplet-film boundary layer thickness is $\ell \sim D_{0} / \sqrt{R e}$.

Since the velocity field inside the boundary layer is unknown, we used scaling analysis to estimate the rate of work done by the viscous forces. The local scales of the fluid velocity $\mathbf{u}_{1}$ and shear stress $\tau_{1}$ in the boundary layer are taken to be $\mathrm{U}_{0}$ and $\mu_{1} \mathrm{~F}_{\mathrm{d}} \mathrm{U}_{0} / \ell$, respectively. 

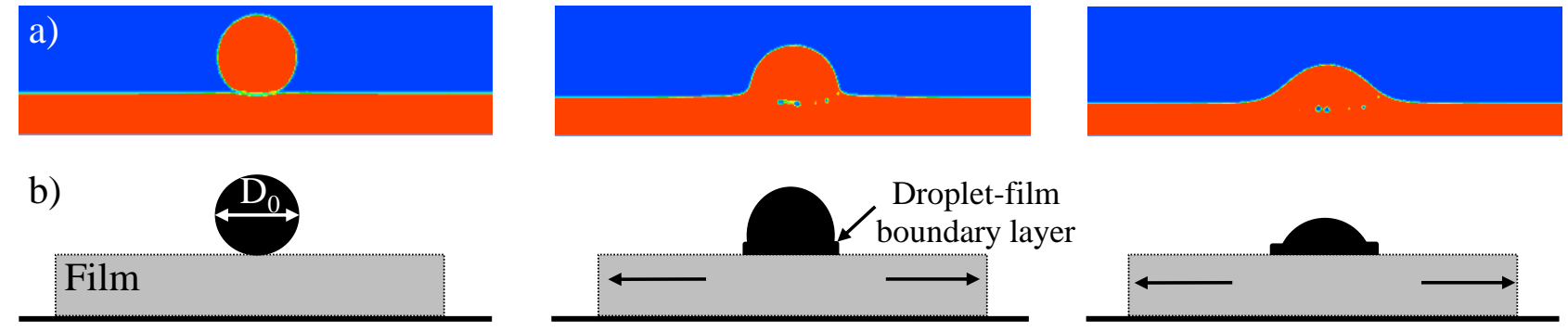

Figure 9: Droplet impingement on a film and merging. a) Volume-Of-Fluid CFD simulation and b) our modeling approach.

We then can write:

$$
\int_{\mathcal{S}_{\mathrm{i}}} \mathbf{n}_{\mathrm{df}} \cdot \tau_{1} \cdot \mathbf{u}_{1} \mathrm{~d} \mathbf{s} \sim-\frac{\mathrm{F}_{\mathrm{d}} \mu_{1} \mathrm{U}_{0}^{2}}{\ell} \mathrm{A}_{\mathrm{i}} \sim-\frac{\mathrm{F}_{\mathrm{d}} \mu_{1} \mathrm{U}_{0}^{2}}{\ell} \frac{\dot{\mathcal{Q}}_{\mathrm{i}}}{\mathrm{U}_{0} \rho_{1}} \sim-\frac{\mathrm{F}_{\mathrm{d}} \mathrm{U}_{0}^{2} \dot{\mathcal{Q}}_{\mathrm{i}}}{\mathrm{Re}} \frac{\mathrm{D}_{0}}{\ell} \sim-\left(\frac{\mathrm{F}_{\mathrm{d}}}{\sqrt{\mathrm{Re}}}\right) \mathrm{U}_{0}^{2} \dot{\mathcal{Q}}_{\mathrm{i}}
$$

where $\mathrm{A}_{\mathrm{i}}$ denotes the area of the surface $\mathcal{S}_{\mathrm{i}}$ (Fig. 8).

Consequently, if $\mathrm{F}_{\mathrm{d}} / \sqrt{\mathrm{Re}}<<1$, the rate of work done by the viscous forces is negligible compared to that done by the pressure forces. This is because:

$$
\left(\frac{\mathrm{F}_{\mathrm{d}}}{\sqrt{\mathrm{Re}}}\right) \mathrm{U}_{0}^{2} \dot{\mathcal{Q}}_{\mathrm{i}}<<\mathrm{U}_{0}^{2} \dot{\mathcal{Q}}_{\mathrm{i}} \Rightarrow \int_{\mathcal{S}_{\mathrm{i}}} \mathbf{n}_{\mathrm{df}} \cdot \tau_{1} \cdot \mathbf{u}_{1} \mathrm{~d} \mathbf{s}<<\int_{\mathcal{S}_{\mathrm{i}}} \mathrm{p}_{1} \mathbf{n}_{\mathrm{df}} \cdot \mathbf{u}_{1} \mathrm{~d} \mathbf{s}
$$

For sprays where $\mathrm{F}_{\mathrm{d}} / \sqrt{\mathrm{Re}} \sim 1$, the mechanical energy balance equation (Equation 20) needs to account for the rate of work done by the viscous forces on each cylindrical film:

$$
\int_{\mathcal{S}_{\mathrm{i}}} \mathbf{n}_{\mathrm{df}} \cdot \tau_{1} \cdot \mathbf{u}_{1} \mathrm{~d} \mathbf{s} \equiv \mathrm{W}_{\mathrm{V}} \sim\left(\frac{\mathrm{F}_{\mathrm{d}}}{\sqrt{\mathrm{Re}}}\right) \mathrm{W}_{\mathrm{P}}
$$

So, by substituting Equations (33) and (36) into (30), we can calculate the overall rate of work done by the surrounding fluid on the surface of each cylindrical film $\left(\mathrm{W}_{\mathrm{S}} \equiv \mathrm{W}_{\mathrm{P}}+\mathrm{W}_{\mathrm{V}}\right)$.

To calculate the thickness $\left(\mathrm{H}_{\mathrm{f}}\right)$ of each disconnected film, one needs to solve the mechanical energy balance Equation (20). To do so, we substituted in it the expressions for the rate of work done by the surrounding fluid on the film surface $\left(\mathrm{W}_{\mathrm{Sf}}\right)$, the rate of change of gravitational, surface and kinetic energies of the film $\left(\mathrm{E}_{\mathrm{Gf}}, \mathrm{E}_{\mathrm{Sf}}, \mathrm{E}_{\mathrm{Kf}}\right)$, as well as the rate of kinetic energy viscous dissipation $\left(\Phi_{\mathrm{f}}\right)$, given by Equations (29), (21), (22), (27) and (28), respectively.

Since the shape of each disconnected film is assumed to be cylindrical $\left[\mathrm{V}(\mathrm{t})=\pi \mathrm{R}_{\mathrm{f}}^{2}(\mathrm{t}) \mathrm{H}_{\mathrm{f}}(\mathrm{t}) ; \mathrm{H}_{\mathrm{m}}=\mathrm{H}_{\mathrm{f}} / 2\right]$, we reduced the number of unknown variables in the mechanical energy balance Equation (20) to one (the variable being $\mathrm{H}_{\mathrm{f}}$ ) and solved it adopting the following initial conditions:

$$
\left.\frac{\mathrm{dR}_{\mathrm{f}}}{\mathrm{dt}}\right|_{\mathrm{t}=\mathrm{t}_{\mathrm{i}}}=\left.\frac{\mathrm{dR} \mathrm{d}_{\mathrm{d}}}{\mathrm{dt}}\right|_{\mathrm{t}=\mathrm{t}_{\mathrm{i}}} ;\left.\quad \mathrm{R}_{\mathrm{f}}\right|_{\mathrm{t}=\mathrm{t}_{\mathrm{i}}}=\left.\mathrm{R}_{\mathrm{d}}\right|_{\mathrm{t}=\mathrm{t}_{\mathrm{i}}}
$$

When the equivalent wetted area $\left(A_{\text {eq }}=N_{d} \pi R_{f}^{2}\right)$ reaches the area of the tablet surface, we assumed that the coating application process is completed. We considered that the final film thickness that covers the tablet is equal to the thickness of each individual film $\mathrm{H}_{\mathrm{f}}$.

To summarize, the current model describes the spreading of disconnected cylindrical coating films under a spray. To simplify the problem we considered that the droplets merge with the film as they impact (Fig. 9) and neglected crown formation [30] and splashing. This means that our model might not yield accurate predictions when these phenomena are relevant. However, it is expected that the effect of these phenomena will be negligible in our case studies where the impinging droplet Weber (We $\equiv \rho_{1} \mathrm{U}_{0}^{2} \mathrm{D}_{0} / \gamma_{12}$ ) and Reynolds numbers are relatively small [12] and droplet-film deposition and merging predominantly occur. 
The model presented allows the estimation of the time $\tau_{F}$ required by the surface of a tablet to become fully covered by a thin coating film. Predicting this "application" time is important, because if the tablet is sprayed for a shorter time period, it will only become partially coated, while if it is sprayed for too long, the coating will spread to adjacent tablets thus possibly leading to inter-tablet coating non-uniformity. In the next section, we present the results of the model and we validate them with experimental data from the literature.

\section{Numerical results and validation}

We validated the numerical results from the model described above with experimental data from the literature. In Section 3.1, we used experimental data from the work of Bolleddula et al. [10] to compare the numerical results concerning single droplet impingement on pharmaceutical tablets. In Section 3.2, we compared the model predictions for the film final thickness with the corresponding experimental results of Kalantari and Tropea [21], who investigated water spray impingement on rigid surfaces while varying impact conditions such as the spray droplet average size and velocity. The wide range of spray impact conditions they investigated and the clarity with which they reported their findings allowed us to validate our model effectively. All the numerical calculations were performed in gPROMS [26], employing the Modelbuilder platform.

It is well documented in the literature that film formation after spray impingement on a rigid surface is influenced by the spray mass flow rate, the droplet mean size and velocity and the liquid properties [11, 21]. We therefore performed variance-based sensitivity analysis employing the gPROMS Modelbuilder global sensitivity analysis tool [26] to study the effect of these parameters on the coating film thickness $\left(\mathrm{H}_{\mathrm{f}}\right)$ and application time $\left(\tau_{\mathrm{F}}\right)$. This analysis indicated how the model output variances depend on the input factors that are subject to uncertainty [31] and allowed us to identify the most important model parameters.

\subsection{Single droplet impact}

In this section, we present the numerical results of the model discussed in Section 2.1 for single droplet impingement on a dry rigid surface. The information derived by this simulation can be used in the prediction of the equivalent wetted area $\left(\mathrm{A}_{\text {eq }}\right)$ for $\mathrm{t}<\mathrm{t}_{\mathrm{i}}$. According to Bolleddula et al. [10], the characteristic time magnitude of the inertia-driven coating spreading regime is approximately equal to $\mathrm{t}_{\mathrm{i}} \equiv \mathrm{D}_{0} / \mathrm{U}_{0}$. This means that for $\mathrm{t}<\mathrm{t}_{\mathrm{i}}$ the droplets spread without the influence of the capillary forces, whose effect is negligible.

In Figure (10.a), we compare our model predictions with the experimental data obtained by Bolleddula et al. [10] for coating droplet impingement (20\% OpadryII White, $\mu_{1}=39.35 \mathrm{cP}, \gamma_{12}=0.04707 \mathrm{~N} / \mathrm{m}$, $\left.\rho_{1}=1040 \mathrm{~kg} / \mathrm{m}^{3}\right)$. The impact velocity and droplet diameter in the experiments and numerical simulations are $2.47 \mathrm{~m} / \mathrm{s}$ and $2.5 \mathrm{~mm}(\mathrm{Re}=163)$, respectively. The experimental measurement errors were reported within $\pm 5 \%$ [10]. The normalized maximum droplet height $\left(\mathrm{h}_{\max } / \mathrm{D}_{0}\right)$ and wetted area diameter $\left(D_{d} / D_{0}\right)$ reach a plateau when the end of the inertia-driven regime is approached (after a time $\mathrm{t} \sim \mathrm{t}_{\mathrm{i}} \equiv \mathrm{D}_{0} / \mathrm{U}_{0} \approx 1 \mathrm{~ms}$ ). Figure (10.a) shows that the model predictions agree well with the experimental data found in the literature.

In Figure (10.b), we show how the kinetic energy of the coating droplet is dissipated after impingement. The droplet kinetic energy approaches zero when the inertia-driven regime is completed at $t=t_{i}$. This means that for longer times $\left(t>t_{i}\right)$ spreading owing to inertial forces would be negligible, if the droplet impinged alone. During spray application, however, the work done on the surface of the wetted film by subsequent droplets makes the film spread further. The numerical results for the spreading owing to successive droplet impacts are discussed in Section 3.2. 

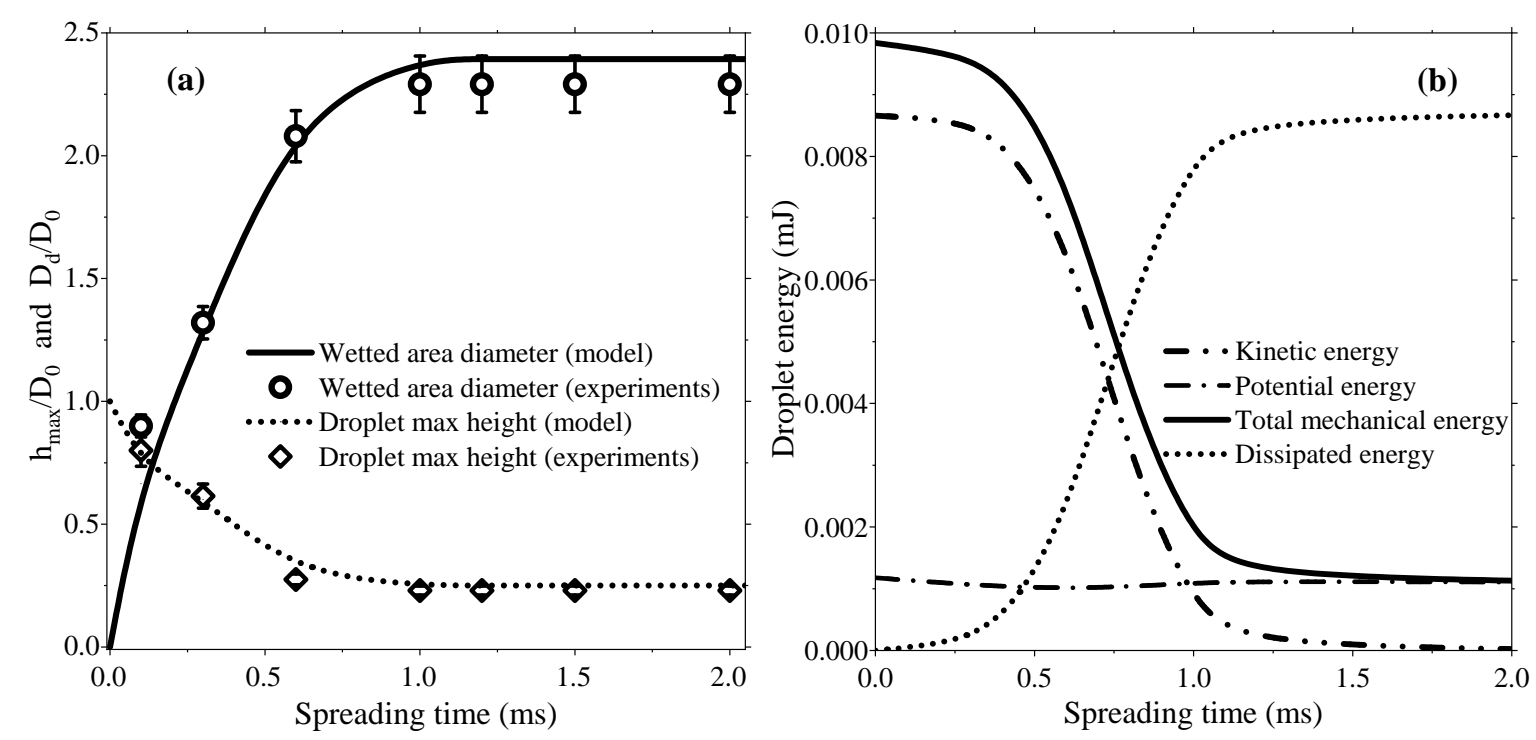

Figure 10: Single droplet impingement on a dry substrate. (Experiments by Bolleddula et al., 2010). a) Normalized wetted area diameter $\left(\mathrm{D}_{\mathrm{d}} / \mathrm{D}_{0}\right)$ and droplet maximum height $\left(\mathrm{h}_{\max } / \mathrm{D}_{0}\right)$.

b) Kinetic energy dissipation during spreading.

\subsection{Spray impact}

In this section, we present the numerical results of the model discussed in Section 2.2 for liquid film spreading after spray impact and we validate them with experimental data found in the literature. Kalantari and Tropea [21] conducted a thorough experimental investigation of water $\left(\rho_{1}=998 \mathrm{~kg} / \mathrm{m}^{3}\right.$, $\mu_{1}=1 \mathrm{cP}, \gamma_{12}=0.072 \mathrm{~N} / \mathrm{m}$ ) spray impact onto a rigid surface. They used a high-speed camera to measure the average film thickness $\left(\overline{\mathrm{H}}_{\mathrm{f}}\right)$ after the "target" surface was completely wetted. It should be noted that in their experiments the thickness $\left(\overline{\mathrm{H}}_{\mathrm{f}}\right)$ did not vary significantly during continuous spraying. In our simulations (Fig. 12), the thickness of each disconnected film increases initially until it reaches a maximum value. After this point, the thickness does not change significantly and each film continues only to spread. We considered that the moment the films come into contact, the "target" surface (in our case the tablet surface) is completely wetted and consequently we calculated $\overline{\mathrm{H}}_{\mathrm{f}}$ at this point.

In Figure (11.a), we compare the experimental data [21] for the normalized film thickness $\left(\overline{\mathrm{H}}_{\mathrm{f}} / \mathrm{D}_{0}\right)$ as a function of the impact Reynolds number with the corresponding numerical results of our model. In our simulations as well as in the experiments, the film thickness $\overline{\mathrm{H}}_{\mathrm{f}}$ was calculated after the entire target surface is wetted (application time, $\mathrm{t}=\tau_{\mathrm{F}}$ ). The water spray volumetric flow rate in both experiments and simulations was set equal to $1 \mathrm{l} / \mathrm{h}\left(\dot{\mathcal{Q}}_{\mathrm{t}}=16.8 \mathrm{~g} / \mathrm{min}\right)$ over a cyclical target $5 \mathrm{~mm}$ in diameter (in our modeling approach this represents the tablet) and the impact Reynolds number was set to range from 300 to $600\left(\mathrm{U}_{0}=7-18 \mathrm{~m} / \mathrm{s}\right.$ and $\left.\mathrm{D}_{0}=30-75 \mu \mathrm{m}\right)$. Kalantari and Tropea [21] reported that the experimental error of the $\overline{\mathrm{H}}_{\mathrm{f}}$ measurements ranged from $5 \mu \mathrm{m}$ to $22 \mu \mathrm{m}$ but they did not mention the corresponding error at each impact condition (impact Re) they investigated. Thus, in Figure (11.a), we introduced $\pm 10 \%$ error bars to assess the agreement between numerical and experimental results. Figure (11.a) shows that our numerical results agree well with the experimental data.

In Figure (11.b), we compare the predictions of our model with the predictions of the empirical equation $\overline{\mathrm{H}}_{\mathrm{f}}=22149 \cdot \mathrm{D}_{0} \operatorname{Re}^{-1.769}$ which was derived by Kalantari and Tropea [21] by fitting their experimental data. As seen in Figure (11.b), the results of our model are in reasonable agreement with the values of the empirical equation $(\mid \%$ Error $\mid<10 \%)$. This is also shown in Table 1, where we calculate the percentage error of the film thickness model predictions for different Re $(\% \mid$ Error $\mid<9.1 \%)$. Even though our model results are close to the empirical equation predictions, the small deviation might be caused by the inability of our model to account for possible splashing and crater formation during droplet-film impact. According to the literature [32], however, the effect of these phenomena should be small in the impact conditions during film-coating (small droplet Reynolds and Weber numbers). This is consistent with the model predictions being good. 

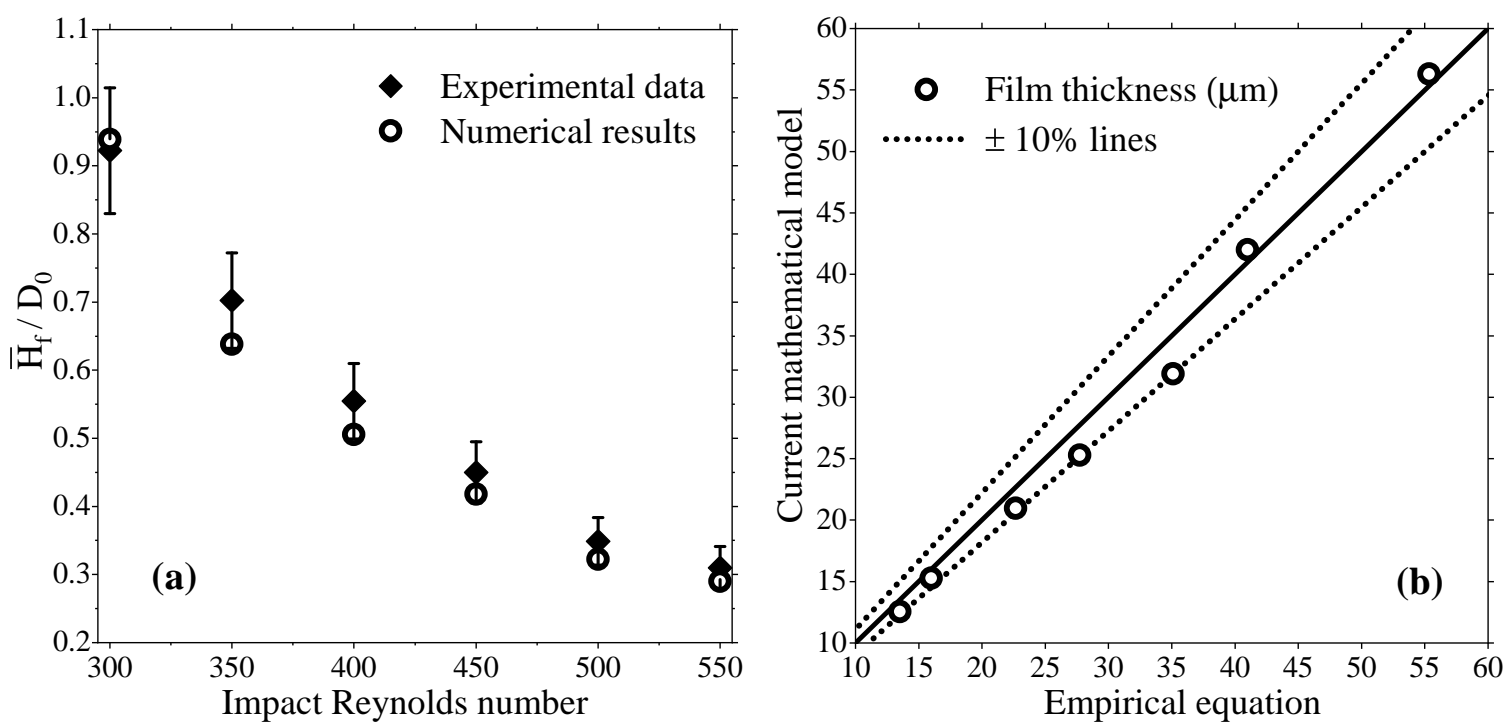

Figure 11: Film height as a function of the droplet impact Reynolds number. Comparison with a) experimental data, and b) empirical equation by Kalantari and Tropea [21].

Table 1: Film thickness at the moment when the entire target surface is wetted $\left(t=\tau_{F}\right)$. Empirical equation by Kalantari and Tropea [21].

\begin{tabular}{cccccc}
\hline $\begin{array}{c}\text { Droplet } \\
\text { diameter }(\mu \mathrm{m})\end{array}$ & $\begin{array}{c}\text { Impact } \\
\text { velocity }(\mathrm{m} / \mathrm{s})\end{array}$ & $\begin{array}{c}\text { Impact } \\
\text { Reynolds number }\end{array}$ & $\begin{array}{c}\text { Film thickness }(\mu \mathrm{m}) \\
\text { Model }\end{array}$ & $\begin{array}{c}\text { Empirical equation } \\
\text { Error } \mid\end{array}$ \\
\hline 60 & 10.0 & 599 & 16.65 & 16.24 & 2.5 \\
65 & 8.0 & 519 & 24.20 & 22.66 & 6.8 \\
55 & 9.0 & 494 & 19.62 & 20.92 & 6.2 \\
30 & 15.0 & 449 & 12.54 & 13.50 & 7.1 \\
50 & 8.0 & 399 & 24.97 & 27.72 & 9.9 \\
\hline
\end{tabular}

The results presented in this section can be used to predict the thickness of the liquid film applied on a tablet surface after passing through the spray zone in a rotating coating drum. If the solvent evaporates evenly from the surface of the tablet then the final polymer film thickness can be estimated.

\subsection{Sensitivity analysis}

It is well documented in the literature that film spreading under spray is affected by many parameters $[11,21]$. These parameters include the size and velocity of the spray droplets before impact, the spray flow rate and liquid viscosity. We performed variance-based sensitivity analysis [31] to assess the influence of these parameters on the model main outputs: the film thickness $\left(\overline{\mathrm{H}}_{\mathrm{f}}\right)$ when the entire tablet surface is wetted and the application time $\left(\tau_{F}\right)$. We used the global sensitivity tool (GSA) of gPROMS Modelbuilder and we followed the method of Saltelli et al. [31] to calculate the first order and total sensitivity indices. The first order sensitivity index, also known as "importance" measure, represents the main effect of the parameter (factor) on the output. The total index additionally accounts for all higher-order effects to the output due to interactions between parameters. A large numerical value indicates a significant effect [26].

Table 2: Sensitivity analysis. Variance-based method by Saltelli [31] in gPROMS Modelbuilder.

\begin{tabular}{lcccc}
\hline Factor & First order $\left(\overline{\mathrm{H}}_{\mathrm{f}}\right)$ & Total effect $\left(\overline{\mathrm{H}}_{\mathrm{f}}\right)$ & First order $\left(\tau_{\mathrm{F}}\right)$ & Total effect $\left(\tau_{\mathrm{F}}\right)$ \\
\hline Droplet diameter, $\mathrm{D}_{0}$ & 0.201 & 0.254 & 0.077 & 0.088 \\
Impact velocity, $\mathrm{U}_{0}$ & 0.467 & 0.526 & 0.170 & 0.189 \\
Spray mass flow rate, $\dot{\mathcal{Q}}$ & 0.001 & 0.059 & 0.635 & 0.641 \\
Liquid viscosity, $\mu_{1}$ & 0.276 & 0.326 & 0.106 & 0.113 \\
\hline
\end{tabular}


From the sensitivity indices in Table 2 we can rank the model parameters based on their effect on the model output (response). The droplet viscosity, velocity and diameter before impact are the parameters that affect the final film thickness $\left(\overline{\mathrm{H}}_{\mathrm{f}}\right)$ the most. The overall spray mass flow rate $(\dot{\mathcal{Q}})$ only has significant secondary effects on the film thickness, but influences significantly the application time $\left(\tau_{\mathrm{F}}\right)$. A small first order effect does not mean that a factor does not affect the response [31], since it might still be important through its interactions with other factors (e.g. $\mathrm{D}_{0}, \mathrm{U}_{0}$ ). This is the reason why we also considered the total effect. The rest of the model parameters (not shown in Table 2) have small sensitivity indices and do not significantly affect the film thickness and application time.

In Figure (12.a), we consider the influence of the droplet impact velocity $\left(\mathrm{U}_{0}\right)$ on the thickness $\left(\mathrm{H}_{\mathrm{f}}\right)$ of the cylindrical films. We present three scenarios for different impact velocities: 4,5 and $6 \mathrm{~m} / \mathrm{s}$. In all scenarios, the droplet mean diameter, the spray mass flow rate and the coating viscosity were set equal to $150 \mu \mathrm{m}, 200 \mathrm{~g} / \mathrm{min}$ and $10 \mathrm{cP}$, respectively. The model predicts that the final value of the film thickness increases when the impact velocity decreases. The simulations also show that the thickness of each film does not vary significantly after it reaches a maximum value. This prediction agrees with experimental observations [13]. At this point, each film continues to spread with the same thickness until the entirety of the tablet surface is wetted. In Figure 12, the application time $\tau_{\mathrm{F}}$ is taken equal to $100 \mathrm{~ms}$ for all case studies.

In Figure (12.b), we study the influence of the spray droplets mean diameter $\left(\mathrm{D}_{0}\right)$ on the film thickness $\left(\mathrm{H}_{\mathrm{f}}\right)$. The velocity of the droplets and the spray rate were set equal to $5 \mathrm{~m} / \mathrm{s}$ and $200 \mathrm{~g} / \mathrm{min}$, respectively. The numerical model predicts that larger droplets form thicker films. Similar behavior is experimentally observed for the single coating droplet impact case; larger droplet diameters result in higher $\mathrm{h}_{\max }$ values [18].
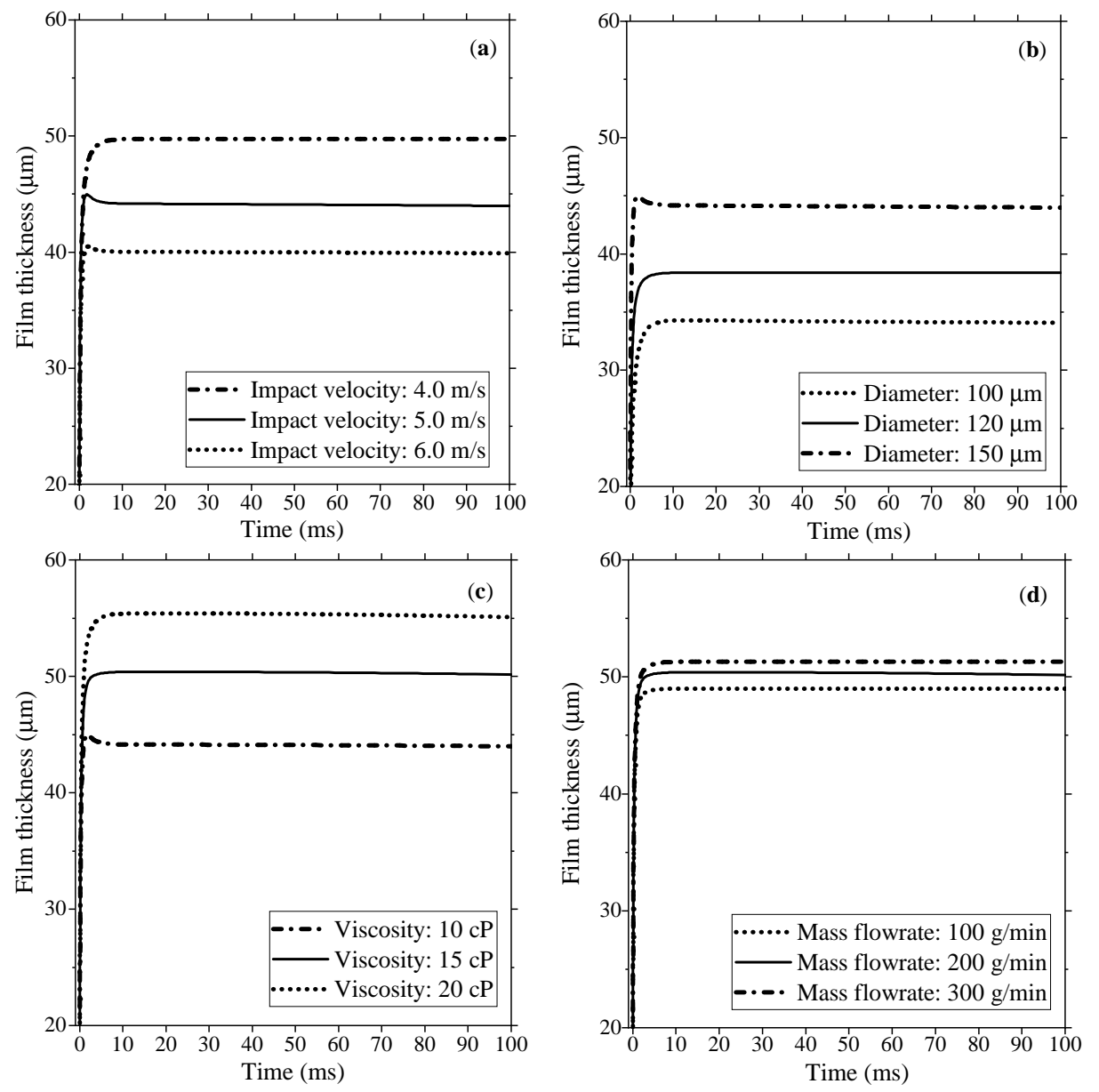

Figure 12: Film $\left(\gamma_{\mathrm{s}}=0.040 \mathrm{~N} / \mathrm{m}, \vartheta_{\mathrm{e}}=30^{\circ}\right)$ thickness variation with changing: a) droplet velocity $\left.\left(\mathrm{D}_{0}=150 \mu \mathrm{m}, \mu_{1}=10 \mathrm{cP}, \dot{\mathcal{Q}}=200 \mathrm{~g} / \mathrm{min}\right), \mathrm{b}\right)$ droplet diameter $\left.\left(\mathrm{U}_{0}=5 \mathrm{~m} / \mathrm{s}, \mu_{1}=10 \mathrm{cP}, \dot{\mathcal{Q}}=200 \mathrm{~g} / \mathrm{min}\right), \mathrm{c}\right)$ viscosity $\left.\left(\mathrm{D}_{0}=150 \mu \mathrm{m}, \dot{\mathcal{Q}}=200 \mathrm{~g} / \mathrm{min}, \mathrm{U}_{0}=5 \mathrm{~m} / \mathrm{s}\right), \mathrm{d}\right)$ spray rate $\left(\mathrm{D}_{0}=150 \mu \mathrm{m}, \mu_{1}=15 \mathrm{cP}, \mathrm{U}_{0}=5 \mathrm{~m} / \mathrm{s}\right)$ 
The importance of the spray liquid properties has often been mentioned in the literature [13]. In Figure (12.c), we show the effect of coating viscosity $\left(\mu_{1}\right)$ on film thickness $\left(\mathrm{H}_{\mathrm{f}}\right)$. The droplet velocity was set at $5 \mathrm{~m} / \mathrm{s}$ and the flow rate at $200 \mathrm{~g} / \mathrm{min}$. As expected, more viscous liquids form thicker films that also spread more slowly. The rate of work done by the viscous forces during droplet-film impact $\left(\mathrm{W}_{\mathrm{V}}\right)$, which is included in the energy balance, is of high significance when concerning highly viscous coatings.

Figure $(12 . \mathrm{d})$ shows the effect of spray mass flow rate $(\dot{\mathcal{Q}})$ on the film thickness $\left(\mathrm{H}_{\mathrm{f}}\right)$. We kept the droplet diameter $(150 \mu \mathrm{m})$ and impact velocity $(5 \mathrm{~m} / \mathrm{s})$ constant, and only changed the number of droplets impinging per unit time (spray density). The numerical simulations show that higher spray densities result in slightly thinner films. The model prediction agrees with the behavior observed in the experiments by Kalantari and Tropea [21].

To summarize, the sensitivity analysis we performed shows that the mean droplet diameter $\left(\mathrm{D}_{0}\right)$ and velocity $\left(\mathrm{U}_{0}\right)$ are the parameters that affect significantly the film thickness and the time required for the coating film to cover the tablet surface which is facing the spray. Moreover, the spray mass flow rate influences mainly the application time and liquid properties such as the coating viscosity $\left(\mu_{1}\right)$ and density $\left(\rho_{1}\right)$ play a less significant role.

\section{Conclusions}

Our work considers the film-coating process, which is widely employed by the pharmaceutical industry. Film coating is a complex process that is difficult to simulate accurately with reasonable computational cost. In this work, we developed a novel mathematical model that can quickly calculate the film thickness and spreading rate on the surface of a tablet passing through the spray zone. The detailed derivations of the main equations are presented in the main article and in the appendix. The results of the model, which was implemented in the gPROMS Modelbuilder platform, were validated with experimental data found in the literature. In Sections 3.1 and 3.2, we showed that the model predictions are in good agreement with the experiments.

Liquid coating film formation during spray impingement is affected by many parameters. To find out which parameters (e.g., film thickness and application time) dominate the output of our mathematical model, we performed a variance-based sensitivity analysis. This analysis was possible because of the short computational time of each simulation (simulation time $<5 \mathrm{~s}$ ), an advantage of our model when compared with traditional CFD models. In Section 3.3, we found that the mean diameter and impact velocity of the droplets are the parameters affecting more strongly the film thickness and the application time. Additionally, we found that the spray mass flow rate significantly influences only the time required to cover the tablet with coating film (application time), whereas liquid properties, such as the coating viscosity and density, are not as important.

Spray properties, such as the atomizing air pressure, the design and configuration of the guns/nozzles and the spray mass flow rate, influence the droplet mean size and velocity, as well as the spray density and spray zone area, attributes that play a key role in controlling the process and enhancing the quality of the tablets. High spray mass flow rates may cause coating defects (the most common being tablet sticking and logo bridging), whereas significantly low spray mass flow rates may lead to spray drying phenomena which affect the duration and efficiency of the coating process [33]. Our model allows predicting the film thickness and application time for a given coating formulation, spray flow rate and droplet mean size and velocity before impact. This insight can be used by the pharmaceutical industry to select the appropriate spray operating conditions and optimize the final product. 


\section{Appendix}

To derive a model that provides solutions for the complex spray impingement problem with relatively short simulation times one needs to make some simplifications/assumptions. In Section 2, we discussed and explained the assumptions made in our model. In this Appendix, we derive the mechanical energy equation used in the model (Section A.1) and we further justify the main assumptions regarding viscous dissipation in the boundary layer (Section A.2), film velocity field (Section A.4), spray splashing, droplet-droplet and drop-film interactions (Sections A.3 and A.5).

\section{A.1 Derivation of the mechanical energy balance equation}

The mechanical energy balance equation in the form of Equation (2) - without the term for the rate of work done by the droplet surroundings - has been used several times in the literature $[17,10]$. However, to the best of our knowledge, no rigorous derivation has ever been reported. Here, we therefore derive the mechanical energy balance equation we employed in the main article.

Consider as control volume the entire droplet. The two surfaces that bound the droplet are dividing surfaces. The first, denoted by $\mathcal{S}_{12}$, is the interface between the liquid and the gas, while the second, denoted by $\mathcal{S}_{13}$, is the interface between the liquid and the solid (Fig. 3). The interface $\mathcal{S}_{23}$ between the gas and the solid is not included in the control volume. The three dividing surfaces meet at the common line $\mathcal{C}$. The three-dimensional spatial region bounded by the dividing surfaces $\mathcal{S}_{12}$ and $\mathcal{S}_{13}$ is denoted by $\mathcal{R}_{1}$. We aim to derive the integral mechanical energy balance equation over the control volume just described. There are various ways of doing this; we base the derivation on the generalized transport theorem, following the approach of Slattery et al. [34]. Let us consider:

$$
\mathrm{D}_{\mathrm{t}} \int_{\mathcal{R}_{1}} \varphi_{1} \mathrm{~d} \mathbf{x}+\mathrm{D}_{\mathrm{t}} \int_{\mathcal{S}_{12}} \varphi_{12} \mathrm{~d} \mathbf{s}+\mathrm{D}_{\mathrm{t}} \int_{\mathcal{S}_{13}} \varphi_{13} \mathrm{~d} \mathbf{s}
$$

where it is:

$$
\varphi_{1} \equiv(1 / 2) \rho_{1} \mathbf{u}_{1} \cdot \mathbf{u}_{1} \quad ; \quad \varphi_{12} \equiv(1 / 2) \rho_{12} \mathbf{u}_{12} \cdot \mathbf{u}_{12} \quad ; \quad \varphi_{13} \equiv(1 / 2) \rho_{13} \mathbf{u}_{13} \cdot \mathbf{u}_{13}
$$

Here $\rho_{\mathrm{r}}$ and $\mathbf{u}_{\mathrm{r}}$ are the fluid density and velocity fields within the region $\mathcal{R}_{\mathrm{r}}$, whereas $\rho_{\mathrm{rs}}$ and $\mathbf{u}_{\mathrm{rs}}$ are the fluid density and velocity fields over the surface $\mathcal{S}_{\mathrm{rs}}$. Using the generalized transport theorem, we now manipulate each term individually. First, we write:

$$
\mathrm{D}_{\mathrm{t}} \int_{\mathcal{R}_{1}} \varphi_{1} \mathrm{~d} \mathbf{x}=\int_{\mathcal{R}_{1}} \partial_{\mathrm{t}} \varphi_{1} \mathrm{~d} \mathbf{x}+\int_{\mathcal{S}_{12}} \mathbf{n}_{12} \cdot \mathbf{u}_{12} \varphi_{1} \mathrm{~d} \mathbf{s}+\int_{\mathcal{S}_{13}} \mathbf{n}_{13} \cdot \mathbf{u}_{13} \varphi_{1} \mathrm{~d} \mathbf{s}
$$

where $\mathbf{n}_{\mathrm{rs}}$ denotes the unit vector normal to $\mathcal{S}_{\mathrm{rs}}$ pointing from phase $\mathrm{r}$ into phase $\mathrm{s}$ (therefore, it is outwardly directed for region $\mathcal{R}_{\mathrm{r}}$ ). For the second integral in Equation (a1), we write:

$$
\mathrm{D}_{\mathrm{t}} \int_{\mathcal{S}_{12}} \varphi_{12} \mathrm{~d} \mathbf{s}=\int_{\mathcal{S}_{12}}\left(\dot{\nabla} \varphi_{12}-2 \mathscr{H}_{12} \mathbf{n}_{12} \cdot \mathbf{u}_{12} \varphi_{12}\right) \mathrm{d} \mathbf{s}+\int_{\mathcal{C}} \mathbf{m}_{12} \cdot \mathbf{u}_{\mathcal{C}} \varphi_{12} \mathrm{~d} \mathbf{s}
$$

Here $\mathscr{H}_{\mathrm{rs}}$ is the mean curvature of the surface $\mathcal{S}_{\mathrm{rs}}$, whereas $\mathbf{m}_{\mathrm{rs}}$ is the unit vector tangent to the surface $\mathcal{S}_{\text {rs }}$, normal to the line $\mathcal{C}$ and outwardly directed. Moreover, $\mathbf{u}_{\mathcal{C}}$ is the fluid velocity field over $\mathcal{C}$ and $\dot{\nabla}$ denotes the invariant time derivative operator $[35,36]$ defined as:

$$
\dot{\nabla} \varphi_{\mathrm{rs}} \equiv \partial_{\mathrm{t}} \varphi_{\mathrm{rs}}-\mathbf{v}_{\mathrm{rs}} \cdot \partial_{\mathrm{s}} \varphi_{\mathrm{rs}}
$$

In this equation, $\partial_{\mathrm{t}} \varphi_{\mathrm{rs}}$ is calculated while holding the surface coordinates constant; therefore, this derivative depends on the parametrization, that is, on the choice of surface coordinates. Also $\mathbf{v}_{\mathrm{rs}}$, which represents the coordinate velocity of the surface (that is, the time rate of change of spatial position following a surface point with fixed surface coordinates), depends on the parametrization. Conversely, the surface gradient $\partial_{\mathrm{s}} \varphi_{\mathrm{rs}}$ and the time derivative $\dot{\nabla} \varphi_{\mathrm{rs}}$ are invariants and do have a clear 
physical meaning. Finally, for the third integral in Equation (a1), we write a similar expression:

$$
\mathrm{D}_{\mathrm{t}} \int_{\mathcal{S}_{13}} \varphi_{13} \mathrm{~d} \mathbf{s}=\int_{\mathcal{S}_{13}}\left(\dot{\nabla} \varphi_{13}-2 \mathscr{H}_{13} \mathbf{n}_{13} \cdot \mathbf{u}_{13} \varphi_{13}\right) \mathrm{d} \mathbf{s}+\int_{\mathcal{C}} \mathbf{m}_{13} \cdot \mathbf{u}_{\mathcal{C}} \varphi_{13} \mathrm{~d} \mathbf{s}
$$

These equations can be manipulated further using the divergence theorem. Let us start with Equation (a3); applying this theorem to the region $\mathcal{R}_{1}$, we obtain the following relation:

$$
\int_{\mathcal{R}_{1}} \partial_{\mathbf{x}} \cdot\left(\mathbf{u}_{1} \varphi_{1}\right) \mathrm{d} \mathbf{x}=\int_{\mathcal{S}_{12}} \mathbf{n}_{12} \cdot \mathbf{u}_{1} \varphi_{1} \mathrm{~d} \mathbf{s}+\int_{\mathcal{S}_{13}} \mathbf{n}_{13} \cdot \mathbf{u}_{1} \varphi_{1} \mathrm{~d} \mathbf{s}
$$

Notice that while in the generalized surface transport theorem the surface integrals feature the velocity of the bounding surface (in Equation a3 these velocities are $\mathbf{u}_{12}$ and $\mathbf{u}_{13}$ ), in the divergence theorem the surface integrals feature the velocity of the fluid evaluated at the boundary of the region - but within the region (in Equation a7 this velocity is $\mathbf{u}_{1}$ ). So, we can write Equation (a3) as follows:

$$
\begin{gathered}
\mathrm{D}_{\mathrm{t}} \int_{\mathcal{R}_{1}} \varphi_{1} \mathrm{~d} \mathbf{x}=\int_{\mathcal{R}_{1}}\left[\partial_{\mathrm{t}} \varphi_{1}+\partial_{\mathbf{x}} \cdot\left(\mathbf{u}_{1} \varphi_{1}\right)\right] \mathrm{d} \mathbf{x}-\int_{\mathcal{S}_{12}} \mathbf{n}_{12} \cdot\left(\mathbf{u}_{1}-\mathbf{u}_{12}\right) \varphi_{1} \mathrm{~d} \mathbf{s}-\int_{\mathcal{S}_{13}} \mathbf{n}_{13} \cdot\left(\mathbf{u}_{1}-\mathbf{u}_{13}\right) \varphi_{1} \mathrm{~d} \mathbf{s} \\
=\int_{\mathcal{R}_{1}}\left[\partial_{\mathrm{t}} \varphi_{1}+\partial_{\mathbf{x}} \cdot\left(\mathbf{u}_{1} \varphi_{1}\right)\right] \mathrm{d} \mathbf{x}-\int_{\mathcal{S}_{12}} \dot{\mathrm{V}}_{12} \varphi_{1} \mathrm{~d} \mathbf{s}-\int_{\mathcal{S}_{13}} \dot{\mathrm{V}}_{13} \varphi_{1} \mathrm{~d} \mathbf{s}
\end{gathered}
$$

where:

$$
\dot{\mathrm{V}}_{12} \equiv \mathbf{n}_{12} \cdot\left(\mathbf{u}_{1}-\mathbf{u}_{12}\right) \quad ; \quad \dot{\mathrm{V}}_{13} \equiv \mathbf{n}_{13} \cdot\left(\mathbf{u}_{1}-\mathbf{u}_{13}\right)
$$

For Equation (a4), we can write:

$$
\int_{\mathcal{S}_{12}} \partial_{\mathbf{s}} \cdot\left(\mathbf{u}_{12} \varphi_{12}\right) \mathrm{d} \mathbf{s}=\int_{\mathcal{C}} \mathbf{m}_{12} \cdot \mathbf{u}_{12} \varphi_{12} \mathrm{~d} \mathbf{s}-\int_{\mathcal{S}_{12}} 2 \mathscr{H}_{12} \mathbf{n}_{12} \cdot \mathbf{u}_{12} \varphi_{12} \mathrm{~d} \mathbf{s}
$$

So, we obtain:

$$
\begin{aligned}
\mathrm{D}_{\mathrm{t}} \int_{\mathcal{S}_{12}} \varphi_{12} \mathrm{~d} \mathbf{s} & =\int_{\mathcal{S}_{12}}\left[\dot{\nabla} \varphi_{12}+\partial_{\mathbf{s}} \cdot\left(\mathbf{u}_{12} \varphi_{12}\right)\right] \mathrm{d} \mathbf{s}-\int_{\mathcal{C}} \mathbf{m}_{12} \cdot\left(\mathbf{u}_{12}-\mathbf{u}_{\mathcal{C}}\right) \varphi_{12} \mathrm{~d} \mathbf{s} \\
& =\int_{\mathcal{S}_{12}}\left[\dot{\nabla} \varphi_{12}+\partial_{\mathbf{s}} \cdot\left(\mathbf{u}_{12} \varphi_{12}\right)\right] \mathrm{d} \mathbf{s}-\int_{\mathcal{C}} \dot{\mathrm{V}}_{12, \mathcal{C}} \varphi_{12} \mathrm{~d} \mathbf{s}
\end{aligned}
$$

With similar passages, we also obtain:

$$
\mathrm{D}_{\mathrm{t}} \int_{\mathcal{S}_{13}} \varphi_{13} \mathrm{~d} \mathbf{s}=\int_{\mathcal{S}_{13}}\left[\dot{\nabla} \varphi_{13}+\partial_{\mathbf{s}} \cdot\left(\mathbf{u}_{13} \varphi_{13}\right)\right] \mathrm{d} \mathbf{s}-\int_{\mathcal{C}} \dot{\mathrm{V}}_{13, \mathcal{C}} \varphi_{13} \mathrm{~d} \mathbf{s}
$$

where:

$$
\dot{\mathrm{V}}_{12, \mathcal{C}} \equiv \mathbf{n}_{12} \cdot\left(\mathbf{u}_{12}-\mathbf{u}_{\mathcal{C}}\right) \quad ; \quad \dot{\mathrm{V}}_{13, \mathcal{C}} \equiv \mathbf{n}_{13} \cdot\left(\mathbf{u}_{13}-\mathbf{u}_{\mathcal{C}}\right)
$$

The results derived permit us to write:

$$
\begin{gathered}
\mathrm{D}_{\mathrm{t}} \int_{\mathcal{R}_{1}} \varphi_{1} \mathrm{~d} \mathbf{x}+\mathrm{D}_{\mathrm{t}} \int_{\mathcal{S}_{12}} \varphi_{12} \mathrm{~d} \mathbf{s}+\mathrm{D}_{\mathrm{t}} \int_{\mathcal{S}_{13}} \varphi_{13} \mathrm{~d} \mathbf{s} \\
=\int_{\mathcal{R}_{1}}\left[\partial_{\mathrm{t}} \varphi_{1}+\partial_{\mathbf{x}} \cdot\left(\mathbf{u}_{1} \varphi_{1}\right)\right] \mathrm{d} \mathbf{x}+\int_{\mathcal{S}_{12}}\left[\dot{\nabla} \varphi_{12}+\partial_{\mathbf{s}} \cdot\left(\mathbf{u}_{12} \varphi_{12}\right)\right] \mathrm{d} \mathbf{s}+\int_{\mathcal{S}_{13}}\left[\dot{\nabla} \varphi_{13}+\partial_{\mathbf{s}} \cdot\left(\mathbf{u}_{13} \varphi_{13}\right)\right] \mathrm{d} \mathbf{s} \\
-\int_{\mathcal{S}_{12}} \varphi_{1} \dot{\mathrm{V}}_{12} \mathrm{~d} \mathbf{s}-\int_{\mathcal{S}_{13}} \varphi_{1} \dot{\mathrm{V}}_{13} \mathrm{~d} \mathbf{s}-\int_{\mathcal{C}}\left(\varphi_{12} \dot{\mathrm{V}}_{12, \mathcal{C}}+\varphi_{13} \dot{\mathrm{V}}_{13, \mathcal{C}}\right) \mathrm{d} \mathbf{s}
\end{gathered}
$$

In our specific problem, there is no mass transfer between the phases; the equation above, consequently, may be simplified. In particular, the last line vanishes, because the velocity of the material particles, of the surface particles and of the common line particles are all equal at the boundaries. Hence, the volume and surface flow rates introduced in Equations (a9) and (a13) are zero. 
We then obtain:

$$
\begin{aligned}
& \mathrm{D}_{\mathrm{t}} \int_{\mathcal{R}_{1}} \varphi_{1} \mathrm{~d} \mathbf{x}+\mathrm{D}_{\mathrm{t}} \int_{\mathcal{S}_{12}} \varphi_{12} \mathrm{~d} \mathbf{s}+\mathrm{D}_{\mathrm{t}} \int_{\mathcal{S}_{13}} \varphi_{13} \mathrm{~d} \mathbf{s}=\int_{\mathcal{R}_{1}}\left[\partial_{\mathrm{t}} \varphi_{1}+\partial_{\mathbf{x}} \cdot\left(\mathbf{u}_{1} \varphi_{1}\right)\right] \mathrm{d} \mathbf{x} \\
& \quad+\int_{\mathcal{S}_{12}}\left[\dot{\nabla} \varphi_{12}+\partial_{\mathbf{s}} \cdot\left(\mathbf{u}_{12} \varphi_{12}\right)\right] \mathrm{d} \mathbf{s}+\int_{\mathcal{S}_{13}}\left[\dot{\nabla} \varphi_{13}+\partial_{\mathbf{s}} \cdot\left(\mathbf{u}_{13} \varphi_{13}\right)\right] \mathrm{d} \mathbf{s}
\end{aligned}
$$

To manipulate this equation further, we integrate over the region $\mathcal{R}_{1}$ the mechanical energy balance equation holding for single-phase fluids [38]. This gives:

$$
\int_{\mathcal{R}_{1}}\left[\partial_{t} \varphi_{1}+\partial_{\mathbf{x}} \cdot\left(\mathbf{u}_{1} \varphi_{1}\right)\right] \mathrm{d} \mathbf{x}=-\int_{\mathcal{R}_{1}}\left[\partial_{\mathbf{x}} \cdot\left(\sigma_{1} \cdot \mathbf{u}_{1}\right)-\tau_{1}: \partial_{\mathbf{x}} \mathbf{u}_{1}-\rho_{1} \mathbf{u}_{1} \cdot \mathbf{g}\right] \mathrm{d} \mathbf{x}
$$

where we have assumed that the fluid is incompressible, so that the field $\mathbf{u}_{1}$ is solenoidal. Here $\sigma_{1}$ is the fluid stress tensor field within region $\mathcal{R}_{1}$ and $\tau_{1}$ is its deviatoric part; $\mathbf{g}$ is the gravitational field. The first term on the right-hand side can be expressed as:

$$
\int_{\mathcal{R}_{1}} \partial_{\mathbf{x}} \cdot\left(\sigma_{1} \cdot \mathbf{u}_{1}\right) \mathrm{d} \mathbf{x}=\int_{\mathcal{S}_{12}} \mathbf{n}_{12} \cdot \sigma_{1} \cdot \mathbf{u}_{1} \mathrm{~d} \mathbf{s}+\int_{\mathcal{S}_{13}} \mathbf{n}_{13} \cdot \sigma_{1} \cdot \mathbf{u}_{1} \mathrm{~d} \mathbf{s}
$$

Moreover, it is:

$$
\int_{\mathcal{R}_{1}} \rho_{1} \mathbf{u}_{1} \cdot \mathbf{g} \mathrm{d} \mathbf{x}=-\int_{\mathcal{R}_{1}} \mathbf{u}_{1} \cdot \partial_{\mathbf{x}} \phi_{1} \mathrm{~d} \mathbf{x}=-\mathrm{D}_{\mathrm{t}} \int_{\mathcal{R}_{1}} \phi_{1} \mathrm{~d} \mathbf{x}
$$

where $\phi_{1}$ is the potential energy per unit volume of the gravitational field in the region considered (as pointed out, this region identifies a material body, because no mass transfer between the phases is present). The last two equations allow writing Equation (a16) in the following form:

$$
\int_{\mathcal{R}_{1}}\left[\partial_{t} \varphi_{1}+\partial_{\mathbf{x}} \cdot\left(\mathbf{u}_{1} \varphi_{1}\right)\right] \mathrm{d} \mathbf{x}=-\mathrm{D}_{\mathrm{t}} \int_{\mathcal{R}_{1}} \phi_{1} \mathrm{~d} \mathbf{x}-\int_{\mathcal{S}_{12}} \mathbf{n}_{12} \cdot \sigma_{1} \cdot \mathbf{u}_{1} \mathrm{~d} \mathbf{s}-\int_{\mathcal{S}_{13}} \mathbf{n}_{13} \cdot \sigma_{1} \cdot \mathbf{u}_{1} \mathrm{~d} \mathbf{s}+\int_{\mathcal{R}_{1}} \tau_{1}: \partial_{\mathbf{x}} \mathbf{u}_{1} \mathrm{~d} \mathbf{x}
$$

We treat the last two terms on the right-hand side of Equation (a15) adopting a similar strategy. Let us integrate over the surface $\mathcal{S}_{12}$ the jump mechanical energy balance equation [34, 37]. Doing so yields the following integral equation:

$$
\begin{gathered}
\int_{\mathcal{S}_{12}}\left[\dot{\nabla} \varphi_{12}+\partial_{\mathbf{s}} \cdot\left(\mathbf{u}_{12} \varphi_{12}\right)\right] \mathrm{d} \mathbf{s}=\int_{\mathcal{S}_{12}}\left[\mathbf{n}_{12} \cdot\left(\sigma_{1}-\sigma_{2}\right) \cdot \mathbf{u}_{12}+2 \mathscr{H}_{12} \mathbf{n}_{12} \cdot \mathbf{u}_{12} \gamma_{12}\right] \mathrm{d} \mathbf{s} \\
+\int_{\mathcal{S}_{12}}\left[\mathbf{u}_{12} \cdot \partial_{\mathbf{s}} \gamma_{12}+\rho_{12} \mathbf{u}_{12} \cdot \mathbf{g}\right] \mathrm{d} \mathbf{s}
\end{gathered}
$$

Here $\sigma_{2}$ is the fluid stress tensor within the region occupied by phase 2, which in our case is the gas, and $\gamma_{12}$ is the surface tension on $\mathcal{S}_{12}$. In writing the equation above, we have assumed that the surface stress tensor is isotropic, so that no viscous part is present. Assuming that $\gamma_{12}$ is uniform (that is, independent of position), the first term on the second line of Equation (a20) vanishes. Moreover, if we assume that the surface densities are constant and uniform, it is:

$$
\begin{gathered}
\int_{\mathcal{S}_{12}} \rho_{12} \mathbf{u}_{12} \cdot \mathbf{g} \mathrm{d} \mathbf{s}=-\int_{\mathcal{S}_{12}} \mathbf{u}_{12} \cdot \partial_{\mathbf{x}} \phi_{12} \mathrm{~d} \mathbf{s}=-\int_{\mathcal{S}_{12}} \mathbf{u}_{12} \cdot\left(\mathbf{I} \cdot \partial_{\mathbf{x}} \phi_{12}\right) \mathrm{d} \mathbf{s} \\
=-\int_{\mathcal{S}_{12}} \mathbf{u}_{12} \cdot\left[\left(\mathbf{n}_{12} \mathbf{n}_{12}+\mathbf{P}\right) \cdot \partial_{\mathbf{x}} \phi_{12}\right] \mathrm{d} \mathbf{s}=-\int_{\mathcal{S}_{12}}\left[\mathbf{u}_{12} \cdot\left(\mathbf{n}_{12} \mathbf{n}_{12} \cdot \partial_{\mathbf{x}} \phi_{12}\right)+\mathbf{u}_{12} \cdot\left(\mathbf{P} \cdot \partial_{\mathbf{x}} \phi_{12}\right)\right] \mathrm{d} \mathbf{s} \\
=-\int_{\mathcal{S}_{12}}\left(\dot{\nabla} \phi_{12}+\mathbf{u}_{12} \cdot \partial_{\mathbf{s}} \phi_{12}\right) \mathrm{d} \mathbf{s}=-\int_{\mathcal{S}_{12}}\left[\dot{\nabla} \phi_{12}+\partial_{\mathbf{s}} \cdot\left(\mathbf{u}_{12} \phi_{12}\right)\right] \mathrm{d} \mathbf{s} \\
=-\int_{\mathcal{S}_{12}}\left(\dot{\nabla} \phi_{12}-2 \mathscr{H}_{12} \mathbf{n}_{12} \cdot \mathbf{u}_{12} \varphi_{12}\right) \mathrm{d} \mathbf{s}-\int_{\mathcal{C}} \mathbf{m}_{12} \cdot \mathbf{u}_{12} \varphi_{12} \mathrm{~d} \mathbf{s}=-\mathrm{D}_{\mathrm{t}} \int_{\mathcal{S}_{12}} \phi_{12} \mathrm{~d} \mathbf{s}
\end{gathered}
$$

where $\mathbf{I}$ and $\mathbf{P}$ are the identity tensor and the projection tensor, respectively, and $\phi_{12}$ is the potential energy per unit surface of the gravitational field over the surface considered. 
Above, in the passage on the third line, we have replaced $\mathbf{u}_{12} \cdot \partial_{\mathbf{s}} \phi_{12}$ with $\partial_{\mathbf{s}}\left(\mathbf{u}_{12} \cdot \phi_{12}\right)$. In general, the two terms differ by the amount $\phi_{12} \partial_{\mathbf{s}} \cdot \mathbf{u}_{12}$. However, because no mass transfer takes place between the phases and because the surface density has been assumed to be constant and uniform, the surface velocity field $\mathbf{u}_{12}$ is solenoidal. To show this, we employ the jump mass balance equation, which reads:

$$
\dot{\nabla} \rho_{12}=\rho_{1} \dot{\mathrm{V}}_{12}+\rho_{2} \dot{\mathrm{V}}_{21}-\partial_{\mathbf{s}} \cdot\left(\rho_{12} \mathbf{u}_{12}\right)
$$

In this equation, because of the assumptions just reported, the invariant time derivative and the mass transfer terms are zero; therefore, so is the divergence of $\rho_{12} \mathbf{u}_{12}$. But since the density is uniform, this implies that the velocity field is divergence-free. This completes the proof.

To manipulate the term in Equation (a20) involving the mean curvature of the surface, we assume that the shape of the droplet is that of a spherical cap. This means that $\mathcal{S}_{13}$ is a disk, whose radius we denote by $R_{d}$, while the common line is a circle. Exploiting the radial symmetry, we can then write:

$$
\begin{gathered}
\int_{\mathcal{S}_{12}} 2 \mathscr{H}_{12} \mathbf{n}_{12} \cdot \mathbf{u}_{12} \gamma_{12} \mathrm{~d} \mathbf{s}=\int_{\mathcal{C}} \mathbf{m}_{12} \cdot \mathbf{u}_{12} \gamma_{12} \mathrm{~d} \mathbf{s}-\mathrm{D}_{\mathrm{t}} \int_{\mathcal{S}_{12}} \gamma_{12} \mathrm{~d} \mathbf{s}=\gamma_{12}\left(\mathbf{m}_{12} \cdot \mathbf{u}_{12} \int_{\mathcal{C}} \mathrm{d} \mathbf{s}-\mathrm{D}_{\mathrm{t}} \int_{\mathcal{S}_{12}} \mathrm{~d} \mathbf{s}\right) \\
=\gamma_{12}\left[\left|\mathbf{u}_{12}\right|\left(2 \pi \mathrm{R}_{\mathrm{d}}\right) \cos \vartheta-\mathrm{D}_{\mathrm{t}} \mathrm{A}_{12}\right]=\gamma_{12}\left[\mathrm{D}_{\mathrm{t}} \mathrm{R}_{\mathrm{d}}\left(2 \pi \mathrm{R}_{\mathrm{d}}\right) \cos \vartheta-\mathrm{D}_{\mathrm{t}} \mathrm{A}_{12}\right] \\
=\gamma_{12}\left[\mathrm{D}_{\mathrm{t}}\left(\pi \mathrm{R}_{\mathrm{d}}^{2}\right) \cos \vartheta-\mathrm{D}_{\mathrm{t}} \mathrm{A}_{12}\right]=\gamma_{12} \mathrm{D}_{\mathrm{t}}\left(\mathrm{A}_{13} \cos \vartheta-\mathrm{A}_{12}\right)
\end{gathered}
$$

where we have assumed that the surface tension is constant (in addition to being uniform). Moreover, $\vartheta$ is the dynamic contact angle - which is not expected to be equal to the equilibrium contact angle, even if one often replaces the former with the latter - whilst $\mathrm{A}_{\mathrm{rs}}$ denotes the area of $\mathcal{S}_{\mathrm{rs}}$. Equation (a23) holds for the case of a cylindrical film where the surface $\mathcal{S}_{13}$ is a disk of radius $\mathrm{R}_{\mathrm{f}}$ (Section 2.2).

The relations derived above allow us to write Equation (a20) in the following form:

$$
\begin{gathered}
\int_{\mathcal{S}_{12}}\left[\dot{\nabla} \varphi_{12}+\partial_{\mathrm{s}} \cdot\left(\mathbf{u}_{12} \varphi_{12}\right)\right] \mathrm{d} \mathbf{s}=-\mathrm{D}_{\mathrm{t}}\left[\int_{\mathcal{S}_{12}} \phi_{12} \mathrm{~d} \mathbf{s}+\gamma_{12}\left(\mathrm{~A}_{12}-\mathrm{A}_{13} \cos \vartheta\right)\right] \\
+\int_{\mathcal{S}_{12}} \mathbf{n}_{12} \cdot\left(\sigma_{1}-\sigma_{2}\right) \cdot \mathbf{u}_{12} \mathrm{~d} \mathbf{s}
\end{gathered}
$$

The last term on the right-hand side of Equation (a15) can be treated similarly; however, since the surface $\mathcal{S}_{13}$ is flat, the term involving the mean curvature vanishes; the final result is:

$$
\int_{\mathcal{S}_{13}}\left[\dot{\nabla} \varphi_{13}+\partial_{\mathbf{s}} \cdot\left(\mathbf{u}_{13} \varphi_{13}\right)\right] \mathrm{d} \mathbf{s}=-\mathrm{D}_{\mathrm{t}} \int_{\mathcal{S}_{13}} \phi_{13} \mathrm{~d} \mathbf{s}+\int_{\mathcal{S}_{13}} \mathbf{n}_{13} \cdot\left(\sigma_{1}-\sigma_{3}\right) \cdot \mathbf{u}_{13} \mathrm{~d} \mathbf{s}
$$

We now introduce the last two equations and Equation (a19) into (a15); doing this yields the integral mechanical energy balance equation:

$$
\begin{gathered}
\mathrm{D}_{\mathrm{t}}\left[\int_{\mathcal{R}_{1}}\left(\varphi_{1}+\phi_{1}\right) \mathrm{d} \mathbf{x}+\int_{\mathcal{S}_{12}}\left(\varphi_{12}+\phi_{12}\right) \mathrm{d} \mathbf{s}+\int_{\mathcal{S}_{13}}\left(\varphi_{13}+\phi_{13}\right) \mathrm{d} \mathbf{s}+\gamma_{12}\left(\mathrm{~A}_{12}-\mathrm{A}_{13} \cos \vartheta\right)\right] \\
=\int_{\mathcal{S}_{12}} \mathbf{n}_{21} \cdot \sigma_{2} \cdot \mathbf{u}_{12} \mathrm{~d} \mathbf{s}+\int_{\mathcal{S}_{13}} \mathbf{n}_{31} \cdot \sigma_{3} \cdot \mathbf{u}_{13} \mathrm{~d} \mathbf{s}+\int_{\mathcal{R}_{1}} \tau_{1}: \partial_{\mathbf{x}} \mathbf{u}_{1} \mathrm{~d} \mathbf{x}
\end{gathered}
$$

The left-hand side features the rate of change of the total energy of the droplet, which comprises kinetic energy, potential energy and surface energy, while the right-hand side features the rate of work performed by phases 2 and 3 on the droplet and the rate of conversion of mechanical energy into internal energy due to the action of the viscous stress within the droplet. 


\section{A.2 Estimation of the rate of viscous dissipation in the droplet-film boundary layer}

In this section, we estimate the rate of viscous dissipation of the droplet kinetic energy in the boundary layer of thickness $\delta$ near the tablet surface (Fig. 4; Section 2.1) where viscous dissipation is significant.

In the boundary layer we do not have a functional form for the velocity field $\mathbf{u}_{1}$. Thus, we cannot solve the following volume integral rigorously:

$$
\Phi_{\widehat{\delta}}=-\int_{\mathcal{R}_{\widehat{\delta}}} \tau_{1}: \partial_{\mathbf{x}} \mathbf{u}_{1} \mathrm{~d} \mathbf{x}
$$

However, we can estimate the value of the rate of viscous dissipation in the boundary layer $\Phi_{\delta}$ using scaling analysis. The rate of viscous dissipation of kinetic energy in the boundary layer region $\mathcal{R}_{\delta}$ is given by:

$$
\begin{gathered}
\Phi_{\bar{\delta}}=-\int_{\mathcal{R}_{\tilde{\delta}}} \tau_{1}: \partial_{\mathbf{x}} \mathbf{u}_{1} \mathrm{~d} \mathbf{x}=\int_{\mathcal{R}_{\tilde{\delta}}}\left[\tau_{\mathrm{rr}} \frac{\partial \mathrm{u}_{1 \mathrm{r}}}{\partial \mathrm{r}}+\tau_{\mathrm{rz}} \frac{\partial \mathrm{u}_{1 \mathrm{r}}}{\partial \mathrm{z}}+\tau_{\vartheta \vartheta}\left(\frac{\mathrm{u}_{1 \mathrm{r}}}{\mathrm{r}}\right)+\tau_{\mathrm{zr}} \frac{\partial \mathrm{u}_{1 \mathrm{z}}}{\partial \mathrm{r}}+\tau_{\mathrm{zz}} \frac{\partial \mathrm{u}_{1 \mathrm{z}}}{\partial \mathrm{z}}\right] \mathrm{d} \mathbf{x} \\
=\int_{\mathcal{R}_{\tilde{\delta}}}\left[2 \mu_{1}\left(\frac{\partial \mathrm{u}_{1 \mathrm{r}}}{\partial \mathrm{r}}\right)^{2}+\mu_{1}\left(\frac{\partial \mathrm{u}_{1 \mathrm{r}}}{\partial \mathrm{z}}+\frac{\partial \mathrm{u}_{1 \mathrm{z}}}{\partial \mathrm{r}}\right) \frac{\partial \mathrm{u}_{1 \mathrm{r}}}{\partial \mathrm{z}}\right. \\
\left.+\mu_{1}\left(\frac{\mathrm{u}_{1 \mathrm{r}}}{\mathrm{r}}\right)^{2}+\mu_{1}\left(\frac{\partial \mathrm{u}_{1 \mathrm{r}}}{\partial \mathrm{z}}+\frac{\partial \mathrm{u}_{1 \mathrm{z}}}{\partial \mathrm{r}}\right) \frac{\partial \mathrm{u}_{1 \mathrm{z}}}{\partial \mathrm{r}}+2 \mu_{1}\left(\frac{\partial \mathrm{u}_{1 \mathrm{z}}}{\partial \mathrm{z}}\right)^{2}\right] \mathrm{d} \mathbf{x}
\end{gathered}
$$

To estimate $\Phi_{\widehat{\delta}}$ we introduce the following local scales:

$$
\frac{\partial \mathrm{u}_{1 \mathrm{r}}}{\partial \mathrm{r}} \sim \frac{\mathrm{v}_{\mathrm{r}}}{\mathrm{R}_{\mathrm{d}}} ; \frac{\partial \mathrm{u}_{1 \mathrm{r}}}{\partial \mathrm{z}} \sim \frac{\mathrm{v}_{\mathrm{r}}}{\delta} ; \frac{\partial \mathrm{u}_{1 \mathrm{z}}}{\partial \mathrm{z}} \sim \frac{\mathrm{v}_{\mathrm{z}}}{\delta}
$$

where $\mathrm{v}_{\mathrm{r}}$ and $\mathrm{v}_{\mathrm{z}}$ are local scales of the radial and vertical velocity in the boundary layer of thickness $\delta$ and $R_{d}$ is the radius of the area of the tablet wetted by the droplet.

We considered that $\partial_{\mathrm{r}} \mathrm{u}_{1 \mathrm{z}}$ is negligible since the changes of the vertical velocity $\left(\mathrm{u}_{1 \mathrm{z}}\right)$ in the radial direction are expected to be very small inside the boundary layer. The boundary layer thickness $\delta$ is given by Equation (5) in Section 2.1. Substituting the scales above into Equation (a28) gives:

$$
\Phi_{\widehat{\delta}} \sim \int_{\mathcal{R}_{\tilde{\delta}}}\left[3 \mu_{1}\left(\frac{\mathrm{v}_{\mathrm{r}}}{\mathrm{R}_{\mathrm{d}}}\right)^{2}+\mu_{1}\left(\frac{\mathrm{v}_{\mathrm{r}}}{\delta}\right)^{2}+2 \mu_{1}\left(\frac{\mathrm{v}_{\mathrm{z}}}{\delta}\right)^{2}\right] \mathrm{d} \mathbf{x}
$$

The first term in the integral on the right-hand side of Equation (a30) can be neglected since: $R_{d}>>\delta$. To relate the scales of the radial and vertical velocities we use the continuity equation:

$$
\frac{1}{\mathrm{r}}\left(\mathrm{r} \frac{\partial \mathrm{u}_{1 \mathrm{r}}}{\partial \mathrm{r}}+\mathrm{u}_{1 \mathrm{r}}\right)+\frac{\partial \mathrm{u}_{1 \mathrm{z}}}{\partial \mathrm{z}}=0 \Rightarrow \frac{\mathrm{v}_{\mathrm{r}}}{\mathrm{R}_{\mathrm{d}}} \sim \frac{\mathrm{v}_{\mathrm{z}}}{\delta} \Rightarrow \mathrm{v}_{\mathrm{z}} \sim \frac{\delta}{\mathrm{R}_{\mathrm{d}}} \mathrm{v}_{\mathrm{r}}
$$

Substituting Equation (a31) into (a30) yields:

$$
\Phi_{\delta} \sim \int_{\mathcal{R}_{\delta}}\left[\mu_{1}\left(\frac{\mathrm{v}_{\mathrm{r}}}{\delta}\right)^{2}+2 \mu_{1}\left(\frac{\mathrm{v}_{\mathrm{r}}}{\mathrm{R}_{\mathrm{d}}}\right)^{2}\right] \mathrm{d} \mathbf{x} \sim \int_{\mathcal{R}_{\delta}} \mu_{1}\left(\frac{\mathrm{v}_{\mathrm{r}}}{\delta}\right)^{2} \mathrm{~d} \mathbf{x} \sim \frac{\mu_{1}}{\delta^{2}} \int_{\mathcal{R}_{\delta}} \mathrm{v}_{\mathrm{r}}^{2} \mathrm{~d} \mathbf{x}
$$

Since the scale of a dependent variable is its maximum value in the region of interest, in the boundary layer we can take $\mathrm{u}_{1 \mathrm{r}}$ to be equal to $\mathrm{v}_{\mathrm{r}}$, where $\mathrm{u}_{1 \mathrm{r}}$ is given by Equation (9). Using the relation $\mathrm{d} \mathbf{x}=\delta \mathrm{d} \mathbf{s}$, denoting the wetted area under the droplet by $\mathcal{S}_{13}$, Equation (a32) becomes:

$$
\Phi_{\delta} \sim \frac{\mu_{1}}{\delta} \int_{\mathcal{S}_{13}} \mathrm{v}_{\mathrm{r}}^{2} \mathrm{~d} \mathbf{s}
$$




\section{A.3 Splashing and droplet-droplet interaction}

In their work, Mundo et al [23] and Bolleddula et al. [10] considered that droplet prompt splashing occurs when:

$$
\mathrm{Oh} \mathrm{Re}^{1.25}>57
$$

where Oh and Re are the Ohnesorge and Reynolds numbers of the droplet upon impact, respectively. In the literature, the empirical criterion (a34) has been used to predict whether splashing occurs when Opadry coating droplets impact on dry tablets [10]. In cases where we have droplet impact on thin films, the criterion is different [11]:

$$
\mathrm{Oh}^{-0.4} \mathrm{We}>2100+5880 \mathrm{~h}^{1.44}
$$

where $\mathrm{h}=\mathrm{H}_{\mathrm{f}} / \mathrm{D}_{0}$ and We is the droplet Weber number at impact. In our work, we studied spray impact where the above criteria $(\mathrm{a} 34$, a35) are both met. However, since the above relations are empirical, further investigation is required to validate them using coating liquids and realistic coating process parameters (droplet velocities and diameters).

It is reported in the literature that droplet-droplet interaction on the surface of a rigid substrate can affect the overall film spreading and lead to secondary droplet generation/splashing [30, 21]. We performed Volume-Of-Fluid simulations (Fig. 13) using the commercial software FLUENT [39] to compare the spreading of two droplets interacting versus a single droplet spreading alone. The droplets in the VOF simulation were $2.5 \mathrm{~mm}$ in diameter $\left(\mathrm{D}_{0}\right)$ and the points of impact were $2 \mathrm{D}_{0}$ apart. In experiments performed by Barnes et al. [22], it was observed that droplet spacing $2 \mathrm{D}_{0}$ results in maximum probability for secondary splashing during droplet-droplet interaction.

\begin{tabular}{|c|c|c|c|c|c|}
\hline \multirow{2}{*}{$\begin{array}{l}\text { Coating } \\
\text { liquid }\end{array}$} & \multirow{2}{*}{$\begin{array}{c}\text { Impact } \\
\text { velocity }(\mathrm{m} / \mathrm{s})\end{array}$} & \multirow{2}{*}{$\begin{array}{c}\text { Liquid } \\
\text { Viscosity }(\mathrm{cP})\end{array}$} & \multicolumn{2}{|c|}{ Wetted area diameter $(\mathrm{mm})$} & \multirow{2}{*}{$\%$ |Error $\mid$} \\
\hline & & & $2 \times$ Single droplet & Two droplets & \\
\hline $70 \%$ Glycerol/Water & 1.0 & 35.5 & $10.10(2 \times 5.05)$ & 10.05 & $0.5 \%$ \\
\hline 15\% OpadryII White PEG & 2.5 & 73.6 & $11.50(2 \times 5.75)$ & 11.15 & $3.0 \%$ \\
\hline 10\% OpadryII White & 5.0 & 98.0 & $11.80(2 \times 5.90)$ & 11.35 & $3.8 \%$ \\
\hline
\end{tabular}

Table 3: Droplet-droplet interaction effect on the wetted area diameter at $t_{i}=D_{0} / U_{0}$.

The results presented in Table 3 show that the droplets collision did not have a significant effect on the overall spreading since the wetted area diameter of the interacting droplets is very close to the wetted area diameter of a single spreading droplet multiplied by two at the same characteristic time $\mathrm{t}_{\mathrm{i}}$. The above results indicate that droplet-droplet interactions can be neglected without significantly sacrificing accuracy in the pharmaceutical coating spraying conditions investigated in this work.

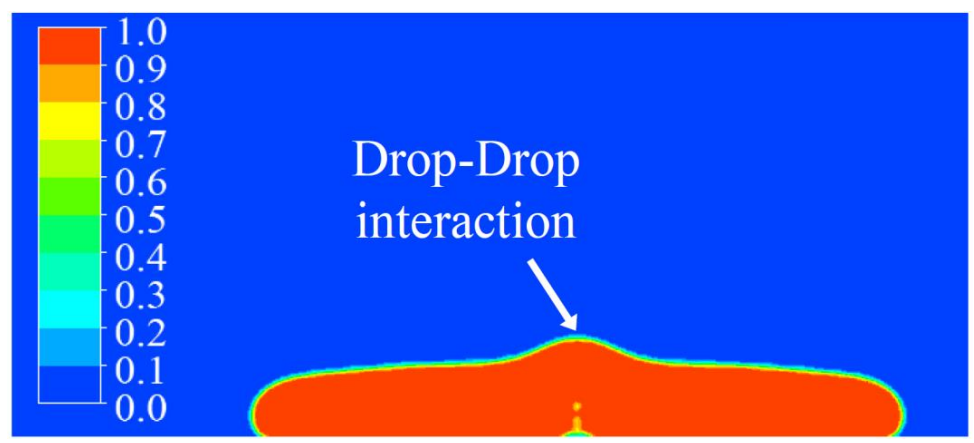

Figure 13: Droplet-droplet interaction on a rigid surface. Volume-Of-Fluid simulation. 


\section{A.4 Cylindrical film velocity field scaling analysis}

In this section, we use scaling analysis to compare the order of magnitude of the velocity field $\mathbf{u}_{1}$ inside the spreading films to that of the droplet impact mean velocity $\left(\mathrm{U}_{0}\right)$. From Equations $(25,26)$ we get:

$$
\begin{gathered}
\mathrm{u}_{1 \mathrm{r}}=\frac{2 \mathrm{rz}}{\mathrm{R}_{\mathrm{f}} \mathrm{H}_{\mathrm{f}}} \frac{\mathrm{dR} \mathrm{R}_{\mathrm{f}}}{\mathrm{dt}} \sim \frac{\mathrm{R}_{\mathrm{f}} \mathrm{H}_{\mathrm{f}}}{\mathrm{R}_{\mathrm{f}} \mathrm{H}_{\mathrm{f}}} \frac{\mathrm{RR}_{\mathrm{f}}}{\mathrm{dt}} \sim \frac{\mathrm{dR}_{\mathrm{f}}}{\mathrm{dt}} \\
\mathrm{u}_{1 \mathrm{z}}=-\frac{2 \mathrm{z}^{2}}{\mathrm{R}_{\mathrm{f}} \mathrm{H}_{\mathrm{f}}} \frac{\mathrm{dR}_{\mathrm{f}}}{\mathrm{dt}} \sim \frac{\mathrm{H}_{\mathrm{f}}^{2}}{\mathrm{R}_{\mathrm{f}} \mathrm{H}_{\mathrm{f}}} \frac{\mathrm{dR}_{\mathrm{f}}}{\mathrm{dt}} \sim \frac{\mathrm{H}_{\mathrm{f}}}{\mathrm{R}_{\mathrm{f}}} \frac{\mathrm{dR}_{\mathrm{f}}}{\mathrm{dt}}
\end{gathered}
$$

To estimate the spreading rate $\left(\mathrm{dR}_{\mathrm{f}} / \mathrm{dt}\right)$ one can assume that when a droplet impinges on the film surface the change in thickness is negligible compared to the wetted area radius increase. Thus, we have:

$$
d V_{f}=2 \pi R_{f} H_{f} d R_{f}=2 \pi R_{f} H_{f} \frac{d R_{f}}{d t} t_{i}=(1 / 6) \pi D_{0}^{3}
$$

where $\mathrm{dV}_{\mathrm{f}}$ is the change of film volume when a droplet impinges on its surface. Since in the simulation case studies investigated in this work we have $\mathrm{H}_{\mathrm{f}} \sim \mathrm{D}_{0}$ and $\mathrm{t}_{\mathrm{i}} \sim \mathrm{D}_{0} / \mathrm{U}_{0}$ we can write:

$$
\mathrm{R}_{\mathrm{f}} \mathrm{D}_{0} \frac{\mathrm{dR}_{\mathrm{f}}}{\mathrm{dt}} \frac{\mathrm{D}_{0}}{\mathrm{U}_{0}} \sim \mathrm{D}_{0}^{3} \Rightarrow \frac{\mathrm{dR}_{\mathrm{f}}}{\mathrm{dt}} \sim \frac{\mathrm{D}_{0}}{\mathrm{R}_{\mathrm{f}}} \mathrm{U}_{0}<<\mathrm{U}_{0}
$$

Substituting Equation (a39) into (a36) and (a37) gives:

$$
\begin{gathered}
\frac{\mathrm{u}_{1 \mathrm{r}}}{\mathrm{U}_{0}} \sim \frac{\mathrm{D}_{0}}{\mathrm{R}_{\mathrm{f}}}<<1 \\
\frac{\mathrm{u}_{1 \mathrm{z}}}{\mathrm{U}_{0}} \sim\left(\frac{\mathrm{D}_{0}}{\mathrm{R}_{\mathrm{f}}}\right)^{2}<<1
\end{gathered}
$$

By comparing the vertical and radial velocity components inside each spreading film we get:

$$
\mathrm{u}_{1 \mathrm{z}}<<\mathrm{u}_{1 \mathrm{r}}<<\mathrm{U}_{0}
$$

Notice that at the very first stages of the spray application, $\mathrm{R}_{\mathrm{f}} \sim \mathrm{H}_{\mathrm{f}} \sim \mathrm{D}_{0}$ and thus the velocity inside the spreading film and the droplet impact velocity $\mathrm{U}_{0}$ have the same order of magnitude. In our simulations, the time interval over which this condition holds is very short compared to that over which the tablet is sprayed. For most of the time we have $D_{0} / R_{f}<<1$ and consequently the order of magnitude of the droplet impact velocity is significantly larger than the one of the velocity field $\mathbf{u}_{1}$ inside each cylindrical film.

\section{A.5 Drop on film}

Equation (31) in Section 2.2 and Equation (a42) in the Appendix Section A.4 suggest that the vertical velocity in the droplet-film boundary layer during merging is significantly smaller than the impact velocity $\left(\mathrm{U}_{0}\right)$. To further support this, we performed CFD Volume-Of-Fluid simulations using FLUENT [39] (Fig.14).

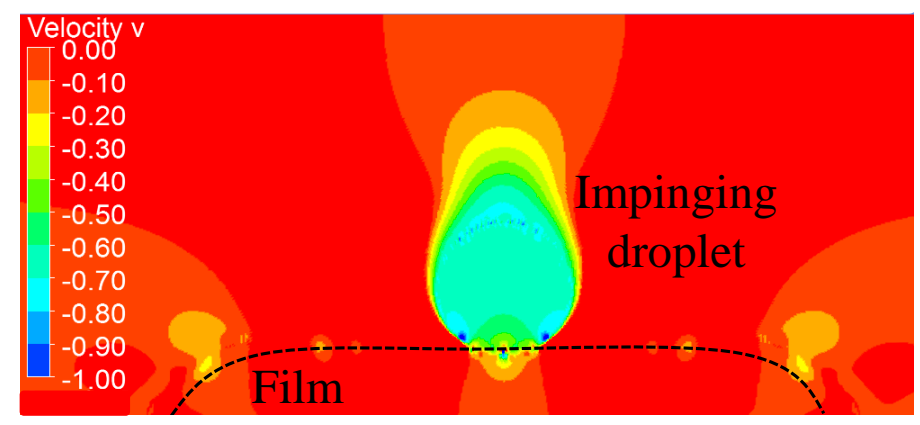

Figure 14: Vertical to the solid substrate velocity component during water droplet impingement on a film $\left(R e=250, U_{0}=1 \mathrm{~m} / \mathrm{s}, D_{0}=2.5 \mathrm{~mm}, \mu_{1}=10 \mathrm{cP}, \rho_{1}=1000 \mathrm{~kg} / \mathrm{m}^{3}\right)$. VOF simulation. 
The results confirm that there is at least an order of magnitude difference between $\mathrm{U}_{0}(\approx 1 \mathrm{~m} / \mathrm{s})$ and the vertical velocity close to the droplet-film interface $\left(\mathrm{u}_{1 \mathrm{z}}<0.1 \mathrm{~m} / \mathrm{s}\right)$. It is therefore reasonable then to assume that at the droplet-film boundary layer the kinetic energy is converted almost entirely into pressure energy and the pressure $\left(\mathrm{p}_{1}\right)$ in Equation (35) can be expressed as: $\mathrm{p}_{1}=(1 / 2) \mathrm{\rho}_{1} \mathrm{U}_{0}^{2}$.

\section{References}

[1] S.C. Porter, Coating of pharmaceutical dosage forms, In: Allen, L.V.E.A., Remington the Science and Practice of Pharmacy, Pharmaceutical Press, London, 977 (2012).

[2] L.A., Felton, Mechanisms of polymeric film formation, International journal of pharmaceutics, 457(2), 423 (2013).

[3] G.C. Cole, Introduction and overview of pharmaceutical coating, Pharmaceutical coating technology, 1, 1 (1995).

[4] G.L. Amidon, P.I Lee, and E.M. Topp, Transport processes in pharmaceutical systems, CRC Press (1999).

[5] A.R. Muliadi, and P.E Sojka, A review of pharmaceutical tablet spray coating, Atomization and Sprays, 20(7), (2010).

[6] A. Aliseda, E.J. Hopfinger, J.C Lasheras, D.M. Kremer, A. Berchielli, and E.K. Connolly, Atomization of viscous and non-Newtonian liquids by a coaxial, high-speed gas jet, Experiments and droplet size modeling, International Journal of Multiphase Flow, 34(2), 161 (2008).

[7] J. Wang, J. Hemenway, W. Chen, D. Desai, W. Early, S. Paruchuri, S.Y. Chang, H. Stamato, and S. Varia, An evaluation of process parameters to improve coating efficiency of an active tablet film-coating process, International journal of pharmaceutics, 427(2), 163 (2012).

[8] M.T. am Ende, and A. Berchielli, A thermodynamic model for organic and aqueous tablet film coating, Pharmaceutical development and technology, 10(1), 47 (2005).

[9] D. Niblett, S. Porter, G. Reynolds, T. Morgan, J. Greenamoyer, R. Hach, S. Sido, K. Karan, and T. Gabbott, Development and evaluation of a dimensionless mechanistic pan coating model for the prediction of coated tablet appearance, International journal of pharmaceutics, 528(1-2), 180 (2017).

[10] D.A. Bolleddula, A. Berchielli and A. Aliseda, Impact of a heterogeneous liquid droplet on a dry surface: Application to the pharmaceutical industry, Advances in colloid and interface science, 159(2), 144 (2010).

[11] G.E. Cossali, M. Marengo, and M. Santini, Single-drop empirical models for spray impact on solid walls: a review, Atomization and Sprays, 15 (2005).

[12] I.V. Roisman, K. Horvat and C. Tropea, Spray Impact: Rim Transverse Instability Initiating Fingering And Splash, And Description Of A Secondary Spray, Physics of Fluids 18,10 (2006).

[13] A.L.N. Moreira, A.S. Moita and M.R Panao, Advances and challenges in explaining fuel spray impingement: How much of single droplet impact research is useful?, Progress in energy and combustion science, 36(5), $554(2010)$.

[14] S.E. Bechtel, D.B. Bogy, and F.E. Talke, Impact of a liquid drop against a flat surface, IBM Journal of Research and Development, 25(6), 963 (1981).

[15] H.Y. Kim, and J.H. Chun, The recoiling of liquid droplets upon collision with solid surfaces, Physics of fluids, 13(3), 643 (2001).

[16] I.V. Roisman, B. Prunet-Foch, C. Tropea, and M. Vignes-Adler, Multiple drop impact onto a dry solid substrate, Journal of colloid and interface science, 256(2), 396 (2002).

[17] P. Attané, F. Girard and V. Morin, An energy balance approach of the dynamics of drop impact on a solid surface. Physics of Fluids, 19,1 (2007).

[18] K.Z.K. Shaari, Coating Uniformity on a Pharmaceutical Tablet: An Experimental Study and Finite Volume Modeling of Droplet Impact Behaviour, PhD thesis, Morgantown, West Virginia, United States (2007). 
[19] C. Christodoulou, E. Sorensen, S. García-Muñoz, and L. Mazzei, Mathematical modelling of water absorption and evaporation in a pharmaceutical tablet during film coating, Chemical Engineering Science, 175,40 (2018).

[20] A.L. Yarin and D.A. Weiss, Impact of drops on solid surfaces: self-similar capillary waves, and splashing as a new type of kinematic discontinuity, Journal of Fluid Mechanics, 283, 141 (1995).

[21] D. Kalantari and C. Tropea, Spray impact onto flat and rigid walls: Empirical characterization and modelling, International Journal of Multiphase Flow, 33(5), 525 (2007).

[22] H.A. Barnes, Y. Hardalupas, A.M.K.P. Taylor and J.H. Wilkins, An investigation of the interaction between two adjacent droplets, ILASS-Europe (1999).

[23] C.H.R. Mundo, M. Sommerfeld and C. Tropea, On the modelling of liquid sprays impinging on surfaces, Atomization and sprays, 8(6), (1998).

[24] S.H. Lee, and H.S. Ryou, Development of a new model and heat transfer analysis of impinging diesel sprays on a wall, Atomization and Sprays, 11(1), (2001).

[25] G.K. Batchelor, An introduction to fluid dynamics, Cambridge university press (2000).

[26] Process Systems Enterprise Ltd. gPROMS User Guide, Release 5.0.2. London, UK, (2018).

[27] J. Madejski, Solidification of droplets on a cold surface, International Journal of Heat and Mass Transfer, 19(9), 1009 (1976).

[28] M. Pasandideh-Fard, Y.M. Qiao, S. Chandra, and J. Mostaghimi, Capillary effects during droplet impact on a solid surface, Physics of fluids, 8(3), 650 (1996).

[29] R. Kumar, B. Freireich, and C. Wassgren, DEM-compartment-population balance model for particle coating in a horizontal rotating drum, Chemical Engineering Science, 125, 144 (2015).

[30] C. Tropea and I.V. Roisman, Modeling of spray impact on solid surfaces, Atomization and sprays, 10(3-5), (2000).

[31] A. Saltelli, P. Annoni, I. Azzini, F. Campolongo, M. Ratto, and S. Tarantola, Variance based sensitivity analysis of model output, Design and estimator for the total sensitivity index. Computer Physics Communications, 181(2), 259 (2010).

[32] A. Bisighini, G.E. Cossali, C. Tropea and I.V. Roisman, Crater evolution after the impact of a drop onto a semi-infinite liquid target, Physical Review E, 82(3), 036319 (2010).

[33] A.M. Agrawal, and P. Pandey, Scale Up of Pan Coating Process Using Quality by Design Principles, Journal of pharmaceutical sciences, 104(11), 3589 (2015).

[34] J. C. Slattery, L. Sagis, and E. Oh, Interfacial Transport Phenomena, Springer (2007).

[35] P. Grinfeld, Introduction to Tensor Analysis and the Calculus of Moving Surfaces, Springer (2013).

[36] P. Cermelli, E. Fried, and M. E. Gurtin, Transport relations for surface integrals arising in the formulation of balance laws for evolving fluid interfaces, Journal of Fluid Mechanics, 544, 339 (2005).

[37] J. M. Delhaye, Jump conditions and entropy sources in two-phase systems. Local instant formulation, International Journal of Multiphase Flow, 1, 395 (1974).

[38] R. B. Bird, W. E. Stewart, and E. N. Lightfoot, Transport Phenomena, Wiley (2007).

[39] Fluent, A.N.S.Y.S., Ansys Fluent, Academic Research, Release, 18 (2017). 